\title{
Global phase portraits of some reversible cubic centers with collinear or infinitely many singularities
}

\author{
M. Caubergh, J. Llibre and J. Torregrosa \\ Departament de Matemàtiques. Edifici C. 08193 Bellaterra (Barcelona) \\ leen@mat.uab.cat, jlibre@mat.uab.cat,torre@mat.uab.cat \\ Received (to be inserted by publisher)
}

\begin{abstract}
We study the reversible cubic vector fields of the form $\dot{x}=-y+a x^{2}+b x y+c y^{2}-y\left(x^{2}+y^{2}\right)$, $\dot{y}=x+d x^{2}+e x y+f y^{2}+x\left(x^{2}+y^{2}\right)$, having simultaneously a center at infinity and at the origin. In this paper the subclass of these reversible systems having collinear or infinitely many singularities are classified with respect to topological and diffeomorphic equivalence.
\end{abstract}

Keywords: center-focus problem, reversible planar vector fields, cubic vector fields, global classification of phase portraits

\section{Introduction}

The center problem for polynomial differential equations in the plane is one of the celebrated and longstanding problems in the qualitative theory of planar differential equations (see e.g. the works [Dulac, 1908], [Kapteyn, 1911], [Kapteyn, 1912], [Bautin, 1954], [Vulpe \& Sibirskiı̌, 1988], [Schlomiuk, 1993], [Żołạdek, 1994a] and [Żołądek, 1994b]). It asks to distinguish a linear center between a focus and a center of a polynomial vector field of a given degree. It is solved completely only for quadratic vector fields; for cubic vector fields it is an open problem. This problem is closely related to another celebrated problem from the beginning of the twentieth century that is still one of the challenges for the twenty first century known as Hilbert's sixteenth problem, which asks essentially for the maximum number $H(n)$ of limit cycles of a polynomial vector field only depending on its degree $n$ (see e.g. [Hilbert, 1902], [Smale, 1998]). Until now even $H(2)$ is not known. Limit cycles, i.e. isolated periodic orbits, can be found for instance by perturbing a center. The mechanism of simultaneous perturbation of different centers is used to construct concrete polynomial vector fields having a certain configuration of limit cycles. As such lower bounds for the socalled Hilbert number $H(n)$ are deduced. For instance in [Shi, 1980] Shi Songling used this mechanism to find that $H(2) \geq 4$; in particular to find the fourth limit cycle for a concrete quadratic vector field an unbounded period annulus ('center at infinity') was involved in the simultaneous bifurcation of quadratic centers.

In [Blows \& Rousseau, 1993] and [Caubergh et al., 2011a] the problem of characterizing a center at the origin and simultaneously at infinity is solved for the following subfamily of the cubic differential equations

$$
\begin{aligned}
& \dot{x}=-y+a x^{2}+b x y+c y^{2}-y\left(x^{2}+y^{2}\right), \\
& \dot{y}=x+d x^{2}+e x y+f y^{2}+x\left(x^{2}+y^{2}\right) .
\end{aligned}
$$

By definition system (1) has a center at infinity if this system after transformation $x=\cos \theta / r, y=\sin \theta / r$ has a center at the origin. It turns out that vector fields of the family (1) that have simultaneously a center at the origin and at infinity are Hamiltonian or reversible. In [Caubergh et al., 2011a] the global 
phase portraits of the Hamiltonian vector fields in the 6-parameter family (1) are analyzed up to $C^{0}$ and $C^{\infty}$-equivalence.

In this paper attention goes to the analysis of the global phase portraits of the reversible vector fields within (1) up to $C^{0}$ and $C^{\infty}$-equivalence. From [Caubergh et al., 2011a] it is known that reversible vector fields in this 6-parameter family of vector fields belong, up to rotation, to the 3-parameter family

$$
X_{(a, b, c)}^{R} \leftrightarrow\left\{\begin{array}{l}
\dot{x}=-y+(a-2 b) x y-y\left(x^{2}+y^{2}\right) \\
\dot{y}=x+c x^{2}+b y^{2}+x\left(x^{2}+y^{2}\right)
\end{array}\right.
$$

where $(a, b, c) \in \mathbb{R}^{3}$. Since the normal form $X_{(a, b, c)}^{R}$ is invariant with respect to the transformation $T_{1}(x, y, t)=(x,-y,-t)$, it follows that the horizontal axis is a line of symmetry; in general any straight line through the origin can occur as line of symmetry by rotation of the horizontal axis about a certain angle.

The main results of this paper are summarized in Theorems 1 and 2.

Theorem 1. Let $X_{(a, b, c)}^{R}$ be a reversible vector field defined in (2). Up to $C^{0}$-equivalence the global phase portraits of $X_{(a, b, c)}^{R}$ having collinear or infinitely many singularities can be classified by the nine phase portraits drawn in Figure 1. For $c \geq 0$ this classification is summarized in the following table:

\begin{tabular}{|c|c|}
\hline$(a, b, c)$ & Figure 1 \\
\hline Case 1: $(2 b-a-c) b<0$ & \multirow{3}{*}{$\begin{array}{l}\text { (I) if } 0 \leq c<2 \\
\text { (IIa) if } c=2 \\
\text { (IIIa) if } c>2 \text { and } 2 b-a-c<0 \\
\text { (IIIc) if } c>2 \text { and } 2 b-a-c>0\end{array}$} \\
\hline Case 2: $b=0$ and $a \neq-c$ & \\
\hline $\begin{array}{cc} & (a-2 b+c) b<0 \\
\text { Case 5: } & \text { and } \\
& (2 b-a)^{2} b /(3 b-a-c)<4 \\
\end{array}$ & \\
\hline Case 3: $b=a+c=0$ & 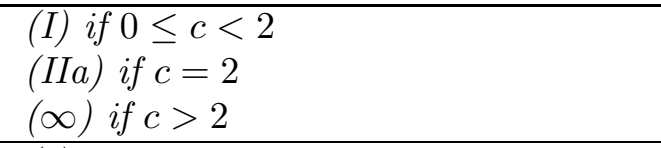 \\
\hline Case $4: a=2 b-c$ and $b \neq 0$ & $\begin{array}{l}\text { (I) if } 0 \leq c<2 \\
\text { (IIa) if } c=2 \text { and } b \leq 1 \\
\text { (IIb) if } c=2 \text { and } b>1 \\
\text { (IIId) if } c>2 \text { and } b<0 \\
\text { (IIIe) if } c>2 \text { and } 0<b \leq b_{-}(c) \\
\text { (IIIa) if } c>2 \text { and } b_{-}(c)<b<b_{+}(c) \\
\text { (IIIb) if } c>2 \text { and } b \geq b_{+}(c)\end{array}$ \\
\hline
\end{tabular}

For $c<0$ the phase portrait of $X_{(a, b, c)}$ is linearly equivalent to the one of $X_{(-a,-b,-c)}$.

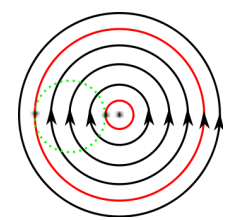

$(\infty)$

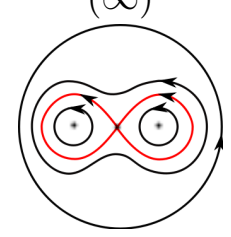

(IIIa)

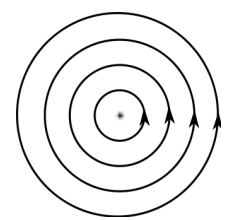

(I)

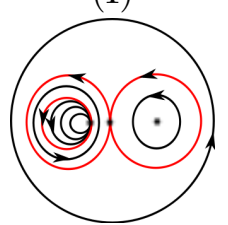

(IIIb)

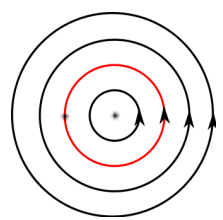

(IIa)

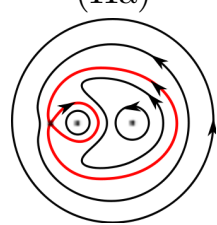

(IIIc)

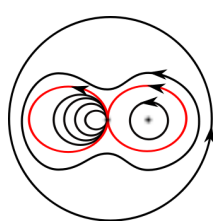

(IIb)

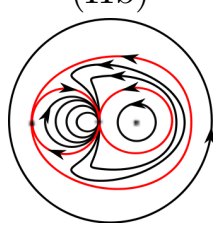

(IIId)

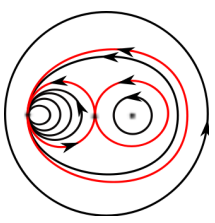

(IIIe)

Fig. 1. Global phase portraits of the cubic reversible vector fields $X_{(a, b, c)}^{R}$ having collinear or infinitely many singularities up to topological equivalence (see Theorem 1). 
From Figure 1 it follows that the phase portraits of $X_{(a, b, c)}^{R}$ with collinear or infinitely many singularities show at most 3 period annuli: 1 in (I), 2 in ( $\infty$ ),(IIa),(IIb),(IIId),(IIIe) and 3 in (IIIa),(IIIb),(IIIc). Except in (IIIb) this number coincides with the total number of centers (with the $\infty$ as a center) present: in (IIIb) there are 3 period annuli present and only 2 centers, the one at $(0,0)$ and the one at $\infty$.

Theorem 2. Let $X_{(a, b, c)}^{R}$ be a reversible vector field defined in (2). The global phase portrait of $X_{(a, b, c)}^{R}$ having collinear or infinitely many singularities can be classified into fifteen distinct phase portraits up to $C^{\infty}$-equivalence. Furthermore for $c \geq 0$ the bifurcation diagram with respect to $C^{\infty}$ equivalence is drawn in

(1) Figure 6 for $b=0$;

(2) Figure 7 for $2 b-a-c \neq 0$;

(3) Figure 15 for $2 b-a-c=0$.

In Figures 1, 2, 6, 7 and 15 attention is paid to the fact that the separatrices have certain contacts, but the exact order of contact they have is not stressed here. The exact order of contact directly follows from the blow up procedure used in the proofs of Propositions 4, 6 and 7.

In particular for the family $\left(X_{(a, b, c)}^{R}\right)$ the $C^{0}$-equivalence class (IIa) (resp. (IIIa)) can be represented by three $C^{\infty}$-equivalence classes: (IIa), (IIa1) and (IIa2)(resp. (IIIa) and (IIIa')). Furthermore for the $C^{0}$-equivalence classes (IIb), (IIIb) in region $\mathcal{R}_{+} \cup \mathcal{B}_{+}$and (IIIe) in region $\mathcal{R}_{-} \cup \mathcal{B}_{-}$there are two different $C^{\infty}$-equivalence classes. On the lines $\mathcal{L}_{ \pm} \equiv\{(a, b, \pm 2): a=2 b-c\}$ the order of contact is higher for $(a, b, c)=( \pm 2, \pm 4, \pm 2)$. On the bifurcation curve $\mathcal{B}_{ \pm}$the order of contact is higher than the one in the region $\mathcal{R}_{ \pm}$, where

$$
\begin{aligned}
\mathcal{B}_{ \pm} & \equiv\left\{(a, b, c):|c|>2, b=\operatorname{sgn}(c) b_{ \pm}(|c|), a=2 b-c\right\}, \\
\mathcal{R}_{+} & \equiv\left\{(a, b, c):|c|>2, b>\operatorname{sgn}(c) b_{+}(|c|), a=2 b-c\right\}, \\
\mathcal{R}_{-} & \equiv\left\{(a, b, c):|c|>2, b<\operatorname{sgn}(c) b_{-}(|c|), a=2 b-c\right\} .
\end{aligned}
$$

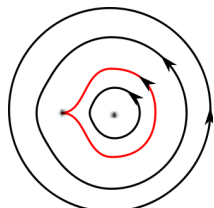

(IIa1)

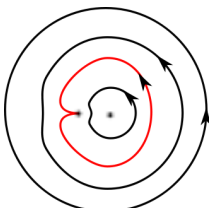

(IIa2)

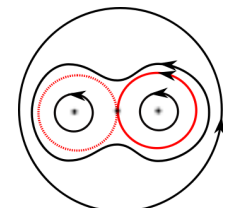

(IIIa')

Fig. 2. Phase portraits (IIa1) and (IIa2) (resp. (IIIa')) are the other representatives with respect to $C^{\infty}$-equivalence contained in the $C^{0}$-equivalence class of (IIa) (resp. (IIIa)) in Figure 1, paying attention to the order of contact of the separatrices (see Theorem 2).

The paper is organized as follows. In section 2 we start by reducing the parameter space $\left\{(a, b, c) \in \mathbb{R}^{3}\right\}$ to $\{c \geq 0\}$ without losing generality. Next we introduce polar coordinates to describe the singularities being collinear or not. From this description we deduce a partition of the parameter space in semi-algebraic sets $\mathcal{P}_{i}, i=1,2,3$, where $\mathcal{P}_{1}$ corresponds to vector fields $X_{(a, b, c)}^{R}$ having infinitely many singularities, $\mathcal{P}_{2}$ corresponds to vector fields $X_{(a, b, c)}^{R}$ having at most 3 singularities which are collinear, and $\mathcal{P}_{3}$ corresponds to vector fields $X_{(a, b, c)}^{R}$ having at most 7 singularities which are noncollinear. In the rest of the paper we only focus on vector fields $X_{(a, b, c)}^{R}$ for which $(a, b, c)$ belongs to $\mathcal{P}_{1} \cup \mathcal{P}_{2}$. For $(a, b, c)$ belonging to $\mathcal{P}_{3}$ an elaborate analysis of the global phase portraits is worked out in [Caubergh et al., 2011b]. Ending section 2 we find that essentially three cases have to be considered in classifying the global phase portraits of $X_{(a, b, c)}^{R}$ when $(a, b, c)$ belongs to $\mathcal{P}_{1} \cup \mathcal{P}_{2}$. 
In particular in section 3 (resp. sections 4 and 5 ) the case is considered when $(a, b, c)$ belongs to $\mathcal{P}_{1}$ (resp. $\mathcal{P}_{2}$ ). In section 4 (resp. 5) the ray carrying singularities is simple (resp. triple). In both sections 4 and 5 we first analyze the local phase portrait near the ray carrying singularities which are more degenerate in section 5. Next by arguments as symmetry, continuity and Poincaré-Bendixson Theorem the classification Theorems 1 and 2 are proven.

\section{Systematic classification}

\subsection{Reduction of the parameter space}

By next lemma we can, without loss of generality, fix the sign of one of the parameters $a, b, c$, when non-zero. In particular in the rest of this paper we will assume that

$$
c \geq 0 .
$$

Lemma 1. Let $(a, b, c) \in \mathbb{R}^{3}$, then the vector field $X_{(a, b, c)}^{R}$ is linearly equivalent to $X_{(-a,-b,-c)}^{R}$.

Proof. The statement follows easily from the fact that the vector field $X_{(a, b, c)}^{R}$ is transformed into $X_{(-a,-b,-c)}^{R}$ by the transformation $T_{2}(x, y, t)=(-x,-y, t)$.

\subsection{Trigonometric functions $A$ and $B$}

In polar coordinates $(r, \theta)$ the family of reversible cubic vector fields in $(2)$ reads as

$$
\begin{aligned}
& r^{\prime}=r^{2} A(\theta), \\
& \theta^{\prime}=1+B(\theta) r+r^{2},
\end{aligned}
$$

where $A$ and $B$ are the following cubic trigonometric polynomials:

$$
\begin{aligned}
& A(\theta)=\sin \theta\left[(a-2 b+c) \cos ^{2} \theta+b \sin ^{2} \theta\right], \\
& B(\theta)=\cos \theta\left[(3 b-a) \sin ^{2} \theta+c \cos ^{2} \theta\right] .
\end{aligned}
$$

In the following lemma we summarize some of their properties that will be used in the classification of the singularities of $X_{(a, b, c)}^{R}$. The proof of this lemma is straight-forward from (5).

Lemma 2. Let $(a, b, c) \in \mathbb{R}^{3}$, let $X_{(a, b, c)}^{R}$ be the reversible vector field defined in (2) and let $A, B$ be the trigonometric functions as defined in (5). Then,

(1) $A(\pi \pm \theta)=\mp A(\theta)$ and $B(\pi \pm \theta)=-B(\theta), \forall \theta \in \mathbb{R}$.

(2) $B(0)=-B(\pi)=c$ and $B(\pi / 2)=0$.

(3) $A(0)=A(\pi)=0$ and $A^{\prime}(0)=a+c-2 b$.

(4) If $a+c-2 b=0$ then $A(0)=A(\pi)=A^{\prime}(0)=A^{\prime \prime}(0)=0$ and $A^{\prime \prime \prime}(\pi)=-6 b$.

(5) If $a+c=b=0$ then $A \equiv 0$.

(6) Suppose that $A$ is not identically zero and $0 \leq \theta^{*} \leq \pi / 2$ is a zero of $A$ (i.e., $A\left(\theta^{*}\right)=0$ ), then

(a) $0<\theta^{*}<\pi / 2$ if and only if $(a-2 b+c) b<0$ and $\cot \theta^{*}=\alpha$ where

$$
\alpha=\sqrt{\left|\frac{b}{a-2 b+c}\right|} .
$$

In particular, $A^{\prime}\left(\theta^{*}\right) \neq 0$.

(b) $A^{\prime}\left(\theta^{*}\right)=0$ and $A^{\prime \prime}\left(\theta^{*}\right) \neq 0$, then $\theta^{*}=\pi / 2$.

(c) $A^{\prime}\left(\theta^{*}\right)=A^{\prime \prime}\left(\theta^{*}\right)=0$, then $\theta^{*}=0$. 


\subsection{Cases}

By Lemma 2 the trigonometric function $A$, if not identically zero, has at most 3 zeroes in $[0, \pi)$ taken into account their multiplicity. Clearly this affects the number of singularities of $X_{(a, b, c)}^{R}$ and their property of being collinear; moreover if $A$ is not identically zero, then $X_{(a, b, c)}^{R}$ has only finitely many singularities. Since $A(0)=A(\pi)=0$ the horizontal ray will carry singularities if $|c| \geq 2$; in particular the multiplicity of $A$ at 0 can only be 1,3 or $\infty$. Since $c \geq 0$ singularities different from the origin only can appear on the negative part of the horizontal axis, i.e. the ray $\mathcal{R}_{0}$ defined by

$$
\mathcal{R}_{0}=\{(x, 0): x<0\} .
$$

In particular we say that the ray $\mathcal{R}_{0}$ (corresponding to $A(\pi)=0$ ) is simple, triple or infinite if respectively

$$
\begin{aligned}
& a+c-2 b \neq 0\left(\Longleftrightarrow A^{\prime}(\pi) \neq 0\right), \\
& a+c-2 b=0\left(\Longleftrightarrow A^{\prime}(\pi)=A^{\prime \prime}(\pi)=0 \text { and } A^{\prime \prime \prime}(\pi) \neq 0\right), \text { or } \\
& b=a+c=0(\Longleftrightarrow A \equiv 0) .
\end{aligned}
$$

Note that, by Lemma 2 , the ray $\mathcal{R}_{0}$ never is double $\left(A^{\prime}(\pi)=0, A^{\prime \prime}(\pi) \neq 0\right)$.

In case the ray $\mathcal{R}_{0}$ is simple (i.e. if $a+c-2 b \neq 0$ ) other rays corresponding to the solution of the trigonometric equation $A\left(\theta^{*}\right)=0,0<\theta^{*} \leq \pi / 2$ are determined by

$$
(a-2 b+c) \cos ^{2} \theta^{*}+b \sin ^{2} \theta^{*}=0 .
$$

If $(a-2 b+c) b>0$ then $\theta^{*}$ is solution of $\cot \theta^{*}= \pm i \alpha$, where $\alpha>0$ is defined in (6); hence $A$ has 1 simple zero and 2 complex ones in $[0, \pi)$. If $(a-2 b+c) b<0$ then $\theta^{*}$ is solution of $\cot \theta^{*}= \pm \alpha$ where $\alpha>0$ is defined in (6); hence in this case $A$ has three simple zeroes in $[0, \pi)$. If $b=0$ and $a+c \neq 0$ then $\theta^{*}=\pi / 2$ is a double zero of $A$. Since furthermore $B(\pi / 2)=0$, the vertical axis does not carry singularities and thus singularities only appear on the horizontal axis. If $b=a+c=0$, then $A \equiv 0$. With respect to the number of zeroes of $A$ and their possible multiplicity the following cases are distinguished, see Figure 3 :

- Case 1: 'One simple - Two complex', i.e. $(a-2 b+c) b>0$

- Case 2: 'One simple - One double', i.e. $b=0$ and $a \neq-c$, corresponding to the 2-parameter family $X_{(a, 0, c)}^{R}, a \neq-c, c \geq 0$.

- Case 3: 'Infinitely many', i.e. $b=a+c=0$, corresponding to the 1-parameter family $X_{(-c, 0, c)}^{R}, c \geq 0$.

- Case 4: 'One triple', i.e. $b \neq 0$ and $a=2 b-c$, corresponding to the 2-parameter family $X_{(2 b-c, b, c)}^{R}, b \neq$ $0, c \geq 0$.

- Case 5: 'Three simple', i.e. $(a-2 b+c) b<0$.

$$
a_{0}<0
$$

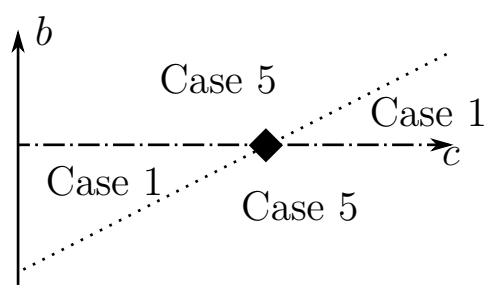

$$
a_{0}=0
$$

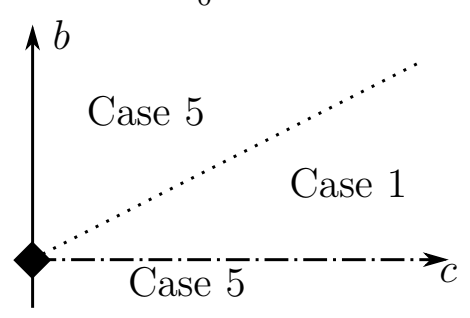

$$
a_{0}>0
$$

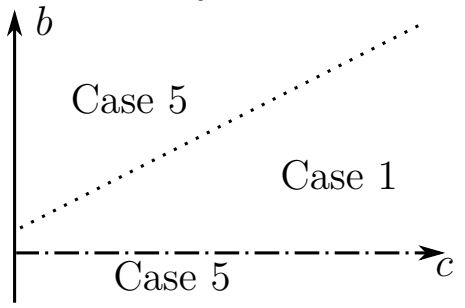

Fig. 3. Regions in the plane $a=a_{0}$ of $(a, b, c)$-space with $c \geq 0$ corresponding to Cases $1,2, \ldots, 5$. The region corresponding to Case 2 (resp. Case 3 and Case 4 ) is indicated by a dash-dotted line (resp. a diamond point and a dotted line).

In this paper we provide with a complete classification of the phase portraits of $X_{(a, b, c)}^{R}$ with collinear or infinitely many singularities. In section 2.4 we will see that in Case 3 there are one, two or infinitely many singularities (see Proposition 1); in Cases 1, 2, 4 and 5 there can be at most finitely many singularities. As we already observed only in Case 5 singularities can appear to be noncollinear. In section 2.4 we will describe a necessary and sufficient condition for the singularities to be collinear in Case 5 (see Proposition 2). For the 
general treatment of Case 5, i.e. not only considering the particular case when all singularities are collinear, it is convenient to introduce new parameters as well as different techniques. An elaborate analysis of Case 5 is organized in a forthcoming paper, see [Caubergh et al., 2011b].

\subsection{Configuration of singularities}

In this section we describe the configurations of the singularities in Cases 1 to 5 distinguishing between cases in which there are finitely and infinitely many. For Case 3 we give a precise bifurcation diagram of the singularities (Proposition 1). In particular, in case there are infinitely many singularities these different from the origin lie on a circle in a half plane $\{x<0\}$. For Cases $1,2,4$ and 5 there are only finitely many singularities and these are in general collinear except in Case 5. For the later case we deduce a necessary and sufficient condition for their singularities being collinear (see Proposition 2). In Proposition 3 we describe the position of the singularities in Cases 1,2, 4 and 5 and distinguish between degenerate and non-degenerate singularities.

Proposition 1. Let $X_{(a, b, c)}^{R}$ be the reversible vector field defined in $(2)$ for $(a, b, c) \in \mathbb{R}^{3}$ with $c \geq 0$ and $a+c=b=0$ (or equivalently $A \equiv 0$ ).

(1) If $0 \leq c<2$ then $X_{(a, b, c)}^{R}$ presents a global center at the origin, linearly equivalent to $\dot{x}=-y, \dot{y}=x$.

(2) If $c=2$ then $X_{(a, b, c)}^{R}$ has exactly two singularities, a center at $(0,0)$ and a singularity with two hyperbolic sectors at $(-1,0)$.

(3) If $c \geq 2$ then $X_{(a, b, c)}^{R}$ has infinitely many singularities, the center at $(0,0)$ and all points on the circle $\mathcal{S}_{c}$ with center at $(-c / 2,0)$ and radius $\sqrt{c^{2}-4} / 2$.

Proof. If $a+c=b=0$ the vector field $X_{(a, b, c)}^{R}$ reads as

$$
\begin{gathered}
\dot{x}=-y\left(1+c x+x^{2}+y^{2}\right), \\
\dot{y}=x\left(1+c x+x^{2}+y^{2}\right) .
\end{gathered}
$$

Therefore $X_{(a, b, c)}^{R}$ has a common factor $1+c x+x^{2}+y^{2}$ and all concentric circles $C_{r}$ with center at the origin and radius $r$ are invariant curves of $X_{(-c, 0, c)}$. Furthermore if $0 \leq c<2$, then the origin of $X_{(-c, 0, c)}$ is a global center linearly equivalent to $\dot{x}=-y, \dot{y}=x$. If $c=2$, then all $C_{r}$ with $r \neq 1$ are regular periodic orbits of $X_{(-2,0,2)}$ and $C_{1}$ is a homoclinic loop with singularity at $(-1,0)$. If $c>2$, then the circle $\mathcal{S}_{c}$ defined by $1+c x+x^{2}+y^{2}=0$ exists of non-isolated singularities of $X_{(-c, 0, c)}$.

Proposition 2. Let $X_{(a, b, c)}^{R}$ be the reversible vector field defined in $(2)$ for $(a, b, c) \in \mathbb{R}^{3}$ with $c \geq 0$ and such that $A$ is not identically zero (or equivalently $(a+c) b \neq 0$ ). Then $X_{(a, b, c)}^{R}$ has only finitely many singularities. In particular these singularities are collinear if one of the following two conditions is satisfied:

$$
(a-2 b+c) b \geq 0 \text { or }\left((a-2 b+c) b<0 \text { and }(2 b-a)^{2} b /(3 b-a-c)<4\right) .
$$

In this case $X_{(a, b, c)}^{R}$ has at most three singularities: the center at the origin and at most two lying on the horizontal ray $\mathcal{R}_{0}$ defined in (7).

Proof. Since $X_{(a, b, c)}^{R}$ has a singularity at the origin and its phase portrait is symmetric with respect to the horizontal axis, its singularities are only collinear when they are situated either at the horizontal or either at the vertical axis. We study this condition further for (2) when written in polar coordinates, taking into account the properties in Lemma 2. Denote the polar coordinates of $\left(x^{*}, y^{*}\right) \neq 0$ by $\left(r^{*}, \theta^{*}\right)$; then $\left(x^{*}, y^{*}\right)$ is a singularity of (2) if and only if $\left(r^{*}, \theta^{*}\right)$ is a singularity of (4), which is equivalent to

$$
A\left(\theta^{*}\right)=0 \text { and } r^{*} \text { positive solution of } 1+B\left(\theta^{*}\right) r+r^{2}=0 .
$$

It hence follows that there are no other singularities than the origin at the vertical axis (since $B(\pi / 2)=$ 0 ) and singularities on the horizontal axis different from the origin lie on $\mathcal{R}_{0}$ (since $B(0)=-B(\pi)=c$ ) for $c \geq 0$. 
Furthermore from Lemma 2 we know that there is at most one $0<\theta^{*}<\pi / 2$ such that $A\left(\theta^{*}\right)=0$; so possible rays for carrying singularities are

$$
\mathcal{R}_{ \pm}^{*} \equiv\left\{\left( \pm r \cos \theta^{*}, r \sin \theta^{*}\right): r>0\right\} \text { and } S\left(\mathcal{R}_{ \pm}^{*}\right),
$$

where $S$ is the reflection with respect to the horizontal axis; hence $S(x, y)=(x,-y)$. If $\left|B\left(\theta^{*}\right)\right|<2$, then there are no singularities on neither of the rays $\mathcal{R}_{ \pm}^{*}$ nor $S\left(\mathcal{R}_{ \pm}^{*}\right)$. If $\left|B\left(\theta^{*}\right)\right| \geq 2$, then there is at least one and there are at most two singularities on $\mathcal{R}_{\sigma}^{*}$ and $S\left(\mathcal{R}_{\sigma}^{*}\right)$ and no singularities on $\mathcal{R}_{-\sigma}^{*} \cup S\left(\mathcal{R}_{-\sigma}^{*}\right)$, where $\sigma=-\operatorname{sgn}\left(B\left(\theta^{*}\right)\right)$.

As a conclusion the singularities of $X_{(a, b, c)}^{R}$ are collinear if and only if $(a-2 b+c) b \geq 0$ or $\left((a-2 b+c) b<0\right.$ and $\left.\left|B\left(\theta^{*}\right)\right|<2\right)$ where $\operatorname{cotan} \theta^{*}=\alpha$ with $\alpha$ as in (6). Since

$$
\left|B\left(\theta^{*}\right)\right|=(2 b-a) \sqrt{b /(3 b-a-c)}<2,
$$

this condition is equivalent to (8).

Let the trace of the Jacobian matrix at a point $(-x, 0) \in \mathcal{R}_{0}$ be denoted by $\operatorname{tr}(J(x))$ and the Jacobian, i.e. the determinant of the jacobian at $(-x, 0)$ by $\operatorname{det}(J(x))$; then

$$
\operatorname{tr}(J(x)) \equiv 0 \text { and } \operatorname{det}(J(x))=(2 b-a-c) x\left(1-x^{2}\right) .
$$

Proposition 3. Let $X_{(a, b, c)}^{R}$ be the reversible vector field defined in $(2)$ for $(a, b, c) \in \mathbb{R}^{3}$ with $c \geq 0$, $(a+c) b \neq 0$ and (8). Then $X_{(a, b, c)}^{R}$ has at most three singularities of which one is the center at the origin and all are collinear and lying on $\mathcal{R}_{0} \cup\{(0,0)\}$ where $\mathcal{R}_{0}$ is defined in (7). Furthermore,

(1) If $0 \leq c<2$, then the ray $\mathcal{R}_{0}$ carries no singularities.

(2) If $c=2$, then the ray $\mathcal{R}_{0}$ carries one singularity, say $\mathbf{s}$ and

$$
\mathbf{s}=(-1,0) .
$$

In particular, $\operatorname{tr}(J(1))=\operatorname{det}(J(1))=0$, and hence the singularity $\mathbf{s}$ is nilpotent or degenerate.

(3) If $c>2$, then the ray $\mathcal{R}_{0}$ carries two singularities, say $\mathbf{s}_{ \pm}$, and

$$
\mathbf{s}_{ \pm}=\left(-x_{ \pm}, 0\right) \text { with } x_{ \pm}=x_{ \pm}(c)=\frac{c \pm \sqrt{c^{2}-4}}{2} .
$$

In particular, $\operatorname{tr}\left(J\left(x_{ \pm}\right)\right) \equiv 0$ and $\operatorname{det}\left(J\left(x_{ \pm}\right)\right)=(2 b-a-c) x_{ \pm}\left(1-x_{ \pm}^{2}\right)$. Therefore,

(a) if $a+c-2 b=0$ then the singularities $\mathbf{s}_{ \pm}$are nilpotent.

(b) if $a+c-2 b>0$ then the singularity $\mathbf{s}_{+}$is a hyperbolic saddle and the singularity $\mathbf{s}_{-}$is a center.

(c) if $a+c-2 b<0$ then the singularity $\mathbf{s}_{+}$is a center and the singularity $\mathbf{s}_{-}$is a hyperbolic saddle.

Proof. This result follows easily from Lemma 2, Proposition 2, the expressions in (9) and the following facts. If $A^{\prime}\left(\theta^{*}\right) \neq 0$ and $r^{*} \neq 1$, then $\mathbf{s}^{*}$ is an elementary singularity of $X_{(a, b, c)}^{R}$. If $\operatorname{det}\left(J\left(\mathbf{s}^{*}\right)\right)<0$, then $\mathbf{s}^{*}$ is a hyperbolic saddle. If $\operatorname{det}\left(J\left(\mathbf{s}^{*}\right)\right)>0$ and $a y^{*}=0$ then $\mathbf{s}^{*}$ is a center. If $\operatorname{det}\left(J\left(\mathbf{s}^{*}\right)\right)>0$ and $a y^{*} \neq 0$ then $\mathbf{s}^{*}$ is a focus or node. If $\operatorname{det}\left(J\left(\mathbf{s}^{*}\right)\right)=0$ but $\operatorname{tr}\left(J\left(\mathbf{s}^{*}\right)\right) \neq 0$, then $\mathbf{s}^{*}$ is a semi-hyperbolic singularity of $X_{(a, b, c)}^{R}$. If $\operatorname{det}\left(J\left(\mathbf{s}^{*}\right)\right)=\operatorname{tr}\left(J\left(\mathbf{s}^{*}\right)\right)=0$ but $J\left(\mathbf{s}^{*}\right) \neq 0$, then $\mathbf{s}^{*}$ is a nilpotent singularity of $X_{(a, b, c)}^{R}$. If $\operatorname{det}\left(J\left(\mathbf{s}^{*}\right)\right)=\operatorname{tr}\left(J\left(\mathbf{s}^{*}\right)\right)=0$ and $J\left(\mathbf{s}^{*}\right)=0$, then $\mathbf{s}^{*}$ is a degenerate singularity of $X_{(a, b, c)}^{R}$.

\subsection{Topological type of semi-hyperbolic and nilpotent singularities}

There exist classification theorems to determine the $C^{0}$ and $C^{\infty}$ type of semi-hyperbolic and nilpotent singularities; we recall them here below in Theorems 3 and 4 in the form they will be used later in this paper. In case of more degenerate singularities we rely on a blow up procedure. A general reference on this theory can be found e.g. in [Andreev, 1958] or [Dumortier et al., 2006] (pp. 92-120). 
Theorem 3 [Semi-Hyperbolic Singular Points Theorem, [Dumortier et al., 2006] pp. 74-75]. Let (0,0) be an isolated singularity of the vector field $X$ given by

$$
\dot{x}=f(x, y), \dot{y}=\mu y+g(x, y)
$$

where $f$ and $g$ are analytic in a neighborhood of $(0,0)$ with

$$
f(0,0)=g(0,0)=0, D f(0,0)=D g(0,0)=0 \text { and } \mu>0 .
$$

Let $y=\mathcal{C}(x)$ be a solution of the equation $\mu y+g(x, y)=0$ in a neighborhood of $(0,0)$ and suppose that the function $F(x)=f(x, \mathcal{C}(x))$ has the expression $F(x)=\rho x^{m}+o\left(x^{m}\right), x \rightarrow 0$ for a non-zero constant $\rho$ and even integer $m \geq 2$. Then there exists an invariant analytic curve tangent at $(0,0)$ to the $y$-axis, on which $X$ is analytically equivalent to $\dot{y}=\mu y$; it represents repelling behavior since $\mu>0$. Furthermore $(0,0)$ is a saddle-node, that is a singularity whose neighborhood is the union of one parabolic and two hyperbolic sectors (see Figure 4). Modulo changing $x$ into $-x$ we suppose that $\rho>0$. Every point to the right of the strong unstable manifold (side $x>0$ ) lies on an invariant $C^{\infty}$ curve, called a center manifold, tangent to the $x$-axis at the origin, and on which $X$ is $C^{\infty}$-conjugate to

$$
\dot{x}=x^{m}\left(1+\beta x^{m-1}\right),
$$

for some $\beta>0$. All these center manifolds coincide on the side $x \leq 0$ and are hence infinitely tangent at the origin. Exactly one of these center manifolds is analytic, in which case $X$ is $C^{\omega}$-conjugate on it to (13). The vector field $X$ is $C^{\infty}$-conjugate to

$$
\dot{x}=x^{m}\left(1+\beta x^{m-1}\right), \dot{y}=\mu y,
$$

and $C^{0}$-conjugate to

$$
\dot{x}=x^{2}, \dot{y}=y
$$

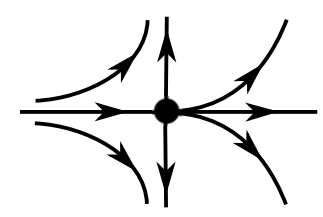

$\rho>0$

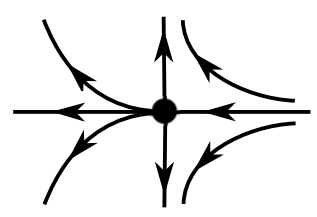

$\rho<0$

Fig. 4. Semi-hyperbolic singularity that is a saddle-node, see Theorem 3.

Theorem 4 [Nilpotent Singular Points Theorem, [Dumortier et al., 2006] pp. 116-117]. Let (0,0) be an isolated singularity of the vector field $X$ given by

$$
\dot{u}=v+f(u, v), \dot{v}=g(u, v),
$$

where $f$ and $g$ are analytic in a neighborhood of $(0,0)$ and also $f(0,0)=g(0,0)$ and $D f(0,0)=D g(0,0)=$ 0 . Let $v=\mathcal{C}(u)$ be a solution of the equation $v+f(u, v)=0$ in a neighborhood of $(0,0)$ and consider $F(u)=g(u, \mathcal{C}(u))$ and $G(u)=\left(\frac{\partial f}{\partial u}+\frac{\partial g}{\partial v}\right)(u, \mathcal{C}(u))$.

(1) If $G(u) \equiv 0$ and $F(u)=\rho u^{m}+o\left(u^{m}\right), u \rightarrow 0$ for a non-zero constant $\rho$ and a positive integer $m \geq 1$, then

(a) if $m$ is even then the singularity is a cusp with direction determined by the sign of $r$; see Figure 5.

(b) if $m$ is odd and $\rho>0$, then the singularity is a saddle.

(c) if $m$ is odd and $\rho<0$, then the singularity is a center or focus.

(2) Suppose that there exists a positive integer $n$ and if there exist non-zero constants $\rho$ and $\sigma$ for which

$$
F(u)=\rho u^{2 n+1}+o\left(u^{2 n+1}\right), G(u)=\sigma u^{n}+o\left(u^{n}\right), u \rightarrow 0 .
$$


(a) If $\rho>0$, then the phase portrait of the origin of $X$ is a saddle point.

(b) If $\delta \equiv \sigma^{2}+4 \rho(n+1)<0$, then the origin of $X$ is a center or a focus.

(c) If $\rho<0$ and $\delta \equiv \sigma^{2}+4 \rho(n+1) \geq 0$, then the phase portrait of the origin of $X$ consists of one hyperbolic and one elliptic sector.

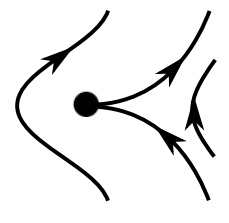

$\rho>0$

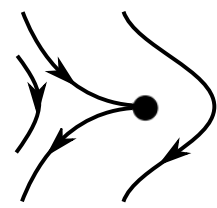

$\rho<0$

Fig. 5. Nilpotent singularity that is a cusp, see Theorem 4.

\section{Global Phase portraits in Case 3: Infinitely many}

Case 3 corresponds to the 1-parameter family $X_{(-c, 0, c)}^{R}, c \geq 0$, or

$$
\begin{gathered}
\dot{x}=-y\left(1+c x+x^{2}+y^{2}\right), \\
\dot{y}=x\left(1+c x+x^{2}+y^{2}\right) .
\end{gathered}
$$

The global phase portrait of $X_{(-c, 0, c)}^{R}$ follows easily from Proposition 1 . Since the vector field $X_{(-c, 0, c)}^{R}$ has the common factor $1+c x+x^{2}+y^{2}$, all concentric circles $C_{r}$ with center at the origin and radius $r$ are invariant curves of $X_{(-c, 0, c)}$. Furthermore if $0 \leq c<2$, then the origin of $X_{(-c, 0, c)}$ is a global center linearly equivalent to $\dot{x}=-y, \dot{y}=x$. If $c=2$, then all $C_{r}$ with $r \neq 1$ are regular periodic orbits of $X_{(-2,0,2)}$ and $C_{1}$ is a homoclinic loop with singularity at $(-1,0)$. If $c>2$, then the circle $\mathcal{S}_{c}$ defined by $1+c x+x^{2}+y^{2}=0$ exists of non-isolated singularities of $X_{(-c, 0, c)}$. Let $r_{-} \equiv\left(c-\sqrt{c^{2}-4}\right) / 2$ and $r_{+} \equiv\left(c+\sqrt{c^{2}-4}\right) / 2$. Then the circles $x^{2}+y^{2}=r_{ \pm}^{2}$ are homoclinic loops with singularity at $\left(-r_{ \pm}, 0\right)$. All circles $x^{2}+y^{2}=$ $r^{2}$ with radius $r<r_{-}$or $r>r_{+}$are regular periodic orbits of $X_{(-c, 0, c)}$. The circles $x^{2}+y^{2}=r^{2}$ with $r_{-}<r<r_{+}$are 2-cycles with singularities at $\left(x_{r}, \pm \sqrt{r^{2}-x_{r}^{2}}\right)$ for $x_{r}=\left(-1-r^{2}\right) / c$.

Notice that the circle $\mathcal{S}_{c}$ has center at $(-c / 2,0)$ and radius $\sqrt{c^{2}-4} / 2$. Therefore the circles $\mathcal{S}_{c}$ grow from the point $(-1,0)$ to an unbounded circle in the left half plane $\{y<0\}$ tangent to the vertical axis $\{y=0\}$ at $(0,0)$ when passing from $c=2$ to $c=+\infty$; that is the centers of $\mathcal{S}_{c}$ move monotonically to the left on the horizontal axis and its radii increase monotonically to $+\infty$ when $c$ increasing to $+\infty$.

The typical phase portrait of $X_{(-c, 0, c)}$ is drawn in Figure 6 in the $(c, a)$-plane.

\section{Global phase portraits when $\mathcal{R}_{0}$ is simple}

In this section we study the global phase portrait of $X_{(a, b, c)}^{R}, c \geq 0$, when the ray $\mathcal{R}_{0}$ defined in (7) is simple, i.e. when $A^{\prime}(\pi)=2 b-a-c \neq 0$. This is the situation in Cases 1, 2 and 5. In particular from Proposition 2 we study $X_{(a, b, c)}^{R}, c \geq 0$ where the parameter $(a, b, c)$ satisfies

$$
(a-2 b+c) b \geq 0 \text { or }\left((a-2 b+c) b<0 \text { and }(2 b-a)^{2} b /(3 b-a-c)<4\right)
$$

and thus singularities only appear on $\mathcal{R}_{0} \cup\{0\}$. At the origin we have a center, and the possible other singularities are described in Proposition 3. We start by detailing the $C^{0}$ and $C^{\infty}$ type of the singularities on the ray $\mathcal{R}_{0}$. Then we will determine the global phase portraits for each of the Cases 1,2 and 5 with respect to $C^{0}$ and $C^{\infty}$ equivalence as stated in Theorems 1 and 2. 


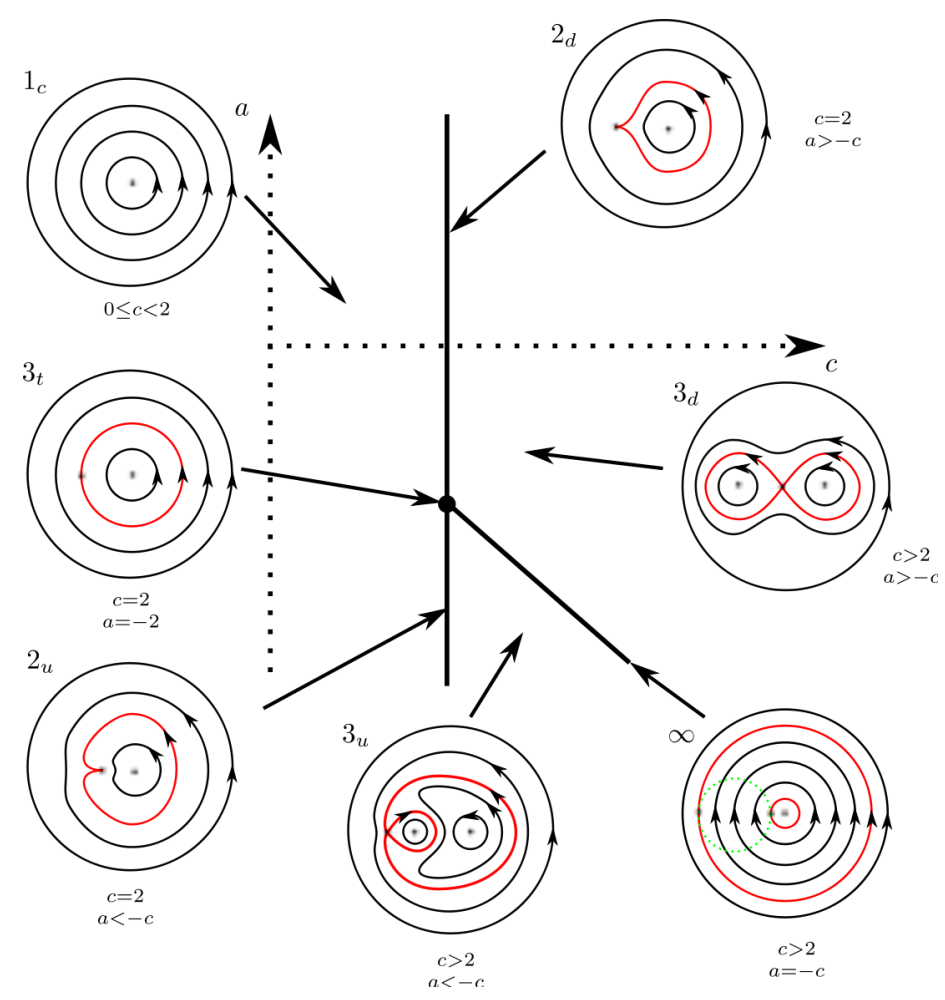

Fig. 6. Global phase portraits of $X_{(a, 0, c)}^{R}$ in the $(c, a)$-plane for $c \geq 0$ (i.e. Cases $2(a \neq-c)$ and $3(a=-c)$ ).

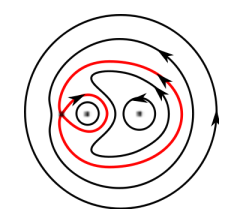

$c>2$

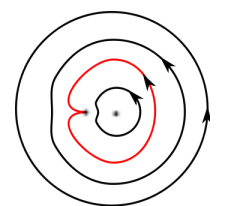

$c=2$

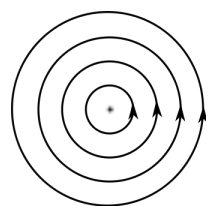

$0 \leq c<2$

I

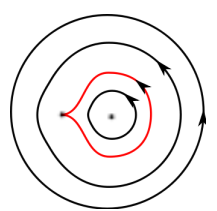

$c=2$

$2 b-a-2<0$

II

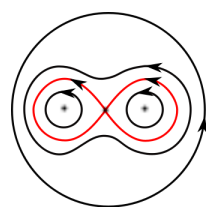

$c>2$

$2 b-a-c<0$

IV

Fig. 7. Global phase portraits in Cases 1,2 and 5 (i.e. $(a-2 b+c) b \geq 0$ or $(a-2 b+c) b<0$ and $\left.(2 b-a)^{2} b /(3 b-a-c)<4\right)$. The roman numbers I, II, III, IV and V refer to the corresponding regions in Figure 9 in Case 1.

\subsection{Local phase portrait along simple ray $\mathcal{R}_{0}$}

Proposition 4. Suppose that $c \geq 0$ and $2 b-a-c \neq 0$. Then for all values of $c \geq 0$ the origin is a center. Depending on the value of $c$ there are one, two or three singularities on the horizontal axis and their type is classified as follows (see Figure 8).

(1) For $0 \leq c<2$ the only singularity on the horizontal axis is the center at the origin.

(2) For $c=2$ there is a cuspidal singularity at $\mathbf{s}=(-1,0)$. Its direction is determined by the sign of $2 b-a-2$.

(3) For $c>2$ there are two other singularities $\mathbf{s}_{ \pm}=\left(-x_{ \pm}, 0\right)$ than the origin where $x_{ \pm}$is defined in (11). If $2 b-a-c>0$ (resp. $2 b-a-c<0$ ) then $\mathbf{s}_{-}$is a center (resp. saddle) and $\mathbf{s}_{+}$is a saddle (resp. center). 


$$
\begin{aligned}
& 0 \leq c<2 \\
& c=2,2 b-a-2>0 \\
& c=2,2 b-a-2<0 \\
& c>2,2 b-a-c>0 \\
& c>2,2 b-a-c<0
\end{aligned}
$$

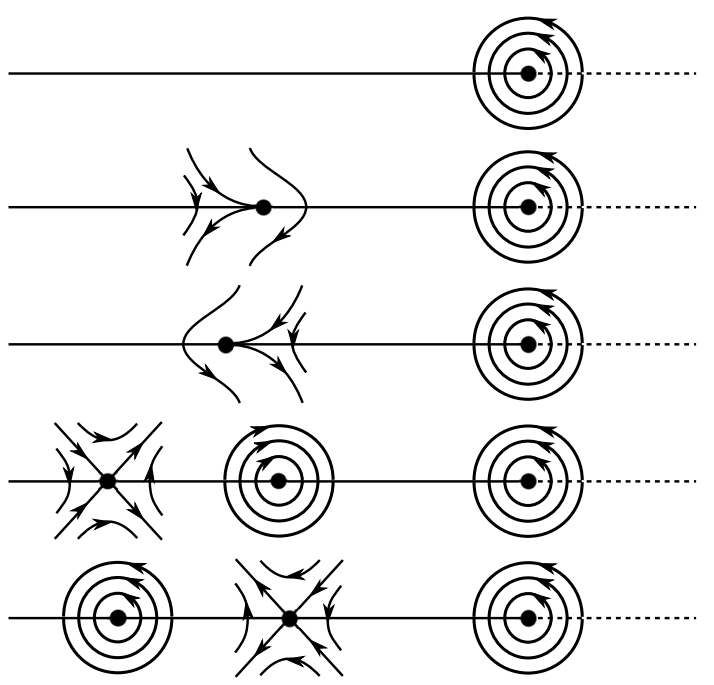

Fig. 8. Local phase portraits near $\mathcal{R}_{0} \cup\{(0,0)\}$ in case $A^{\prime}(0) \neq 0$, see Proposition 4 .

Proof. Because $A(\pi)=0$ and $B(\pi)=-c$ we find resp. 1, 2 and 3 singularities on the negative horizontal axis for $0 \leq c<2, c=2$ and $c>2$. The Jacobian at $(-r, 0)$ for $r>0$ can be written as

$$
\operatorname{det} J(r)=r(2 b-a-c)(2-c r) \text {. }
$$

For $c>2$ we have two singularities for $r=x_{ \pm}$and it can be checked that $2-c x_{-}>0$ and $2-c x_{+}<0$. If the Jacobian $\operatorname{det} J\left(x_{ \pm}\right)$is negative then the corresponding singularity is a saddle point. If the Jacobian is positive then the singularity is a center by the symmetry with respect to the $x$-axis. Therefore the result follows for $c>2$.

For $c=2$ the determinant of $J(r)$ vanishes since then $r=1$. In fact

$$
J(r)=\left(\begin{array}{cc}
0 & 2 b-a-2 \\
0 & 0
\end{array}\right),
$$

therefore we have a nilpotent singularity at $(-1,0)$ for $c=2$. After translation $(x, y) \mapsto(x+1, y)$ and division by $(2 b-a-2)$ the vector field $X_{(a, b, 2)}$ is transformed in the equivalent one of type (14) with

$$
f(x, y)=-y\left(x+\frac{x^{2}+y^{2}}{2 b-a-2}\right) \text { and } g(x, y)=\frac{-x^{2}-y^{2}+b y^{2}+x y^{2}+x^{3}}{2 b-a-2} .
$$

Notice that for $2 b-a-2>0$ (resp. $<0$ ) time is preserved (resp. reversed). The functions $\mathcal{C}, F, G$ in Theorem 4 now are

$$
\mathcal{C}(x) \equiv 0, F(x)=\frac{x^{3}-x^{2}}{2 b-a-2} \text { and } G(x)=\left.\frac{a y}{2 b-a-2}\right|_{y=0} \equiv 0 .
$$

Therefore by Theorem 4 the singularity $(-1,0)$ is a cusp and its direction is as in Figure $5(\rho<0)$ if $2 b-a-2>0$ and as in Figure $5(\rho>0)$ with time reversed if $2 b-a-2<0$.

\subsection{Case 1: One simple - two complex}

In this case the parameter $(a, b, c)$ satisfies $c \geq 0$ and $(a-2 b+c) b>0$, or equivalently $c \geq 0$ and $(0<2 b<a+c$ or $a+c<2 b<0)$.

The local phase portrait along the ray $\mathcal{R}_{0}$ is established in Proposition 4 . As we noticed before all possible singularities are located along $\mathcal{R}_{0} \cup\{0\}$ (since $A(\theta)=0$ for $\theta \in[0, \pi$ ) if and only if $\theta=0$ and $c \geq 0$ ). A local bifurcation happens when passing through $c=2$ as this corresponds to the appearance of a singularity for $c \uparrow 2$ and the collision of two singularities into one for $c \downarrow 2$. Therefore in Case 1 we distinguish between the following regions in the parameter space $(a, b, c)$, see Figure 9: 
- Region I: $0 \leq c<2$,

- Region II: $c=2$ and $0<2 b<a+c$,

- Region III: $c=2$ and $a+c<2 b<0$,

- Region $I V: c>2$ and $0<2 b<a+c$,

- Region $V: c>2$ and $a+c<2 b<0$.

Starting from the local phase portrait near the ray $\mathcal{R}_{0} \cup\{0\}$, the global phase portrait can be completed using the symmetry property of $X_{(a, b, c)}^{R}$ with respect to the $x$-axis. In Figure 7 the corresponding global phase portraits of $X_{(a, b, c)}^{R}$ are described. For Region I $(0 \leq c<2)$ the origin is a global center. For $c=2$ we have a homoclinic loop connecting a cuspidal singularity at $(-1,0)$; the direction of the cusp is determined by the sign of $b$. For Region II (resp. Region III) the cusp is pointing outwards (resp. inwards). For $c \geq 2$ we have a double homoclinic loop at a hyperbolic saddle point enclosing two centers. For Region IV (resp. Region V) there is a saddle (resp. center) at $\left(\left(-c+\sqrt{c^{2}-4}\right) / 2,0\right)$ and a center (resp. saddle) at $\left(\left(-c-\sqrt{c^{2}-4}\right) / 2,0\right)$.
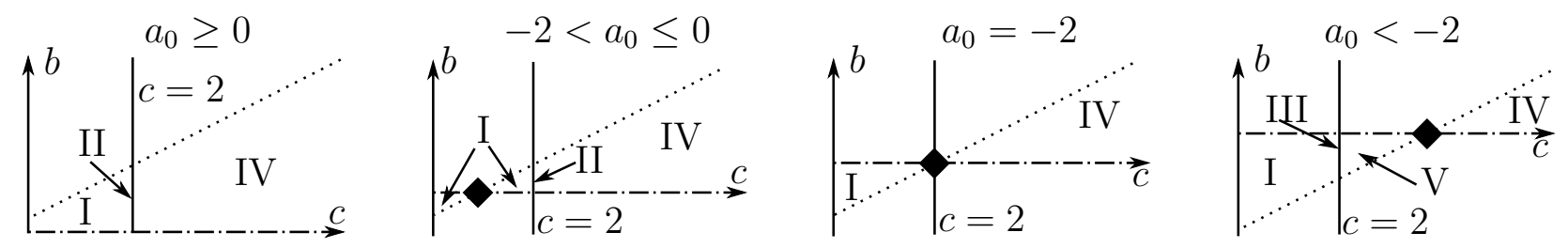

Fig. 9. Bifurcation diagram of global phase portraits of $X_{(a, b, c)}^{R}$ in the plane $\left\{a=a_{0}\right\}$ in the $(a, b, c)$-space restricting to $c \geq 0$ and $(a-2 b+c) b>0$ (i.e. Case 1). The phase portraits are drawn in Figure 7.

\subsection{Case 2: One simple - one double}

In this case the parameter $(a, b, c)$ satisfies $c \geq 0, b=0$ and $a \neq-c$ and we are left with the 2-parameter family of reversible cubic vector fields $X_{(a, 0, c)}^{R}$. Then the trigonometric functions $A$ resp. $B$ read as

$$
\begin{aligned}
& A(\theta)=(a+c) \sin \theta \cos ^{2} \theta, \\
& B(\theta)=\cos \theta\left[-a \sin ^{2} \theta+c \cos ^{2} \theta\right] .
\end{aligned}
$$

Clearly $A(\theta)=0$ for $\theta \in[0, \pi)$ if and only if $\theta=0$ or $\theta=\pi / 2$ (see Lemma 2 and Proposition 3); furthermore $B(0)=c$ and $B(\pi / 2)=0$. Hence $X_{(a, 0, c)}$ only can have singularities on the negative horizontal axis $\mathcal{R}_{0}$ which is simple. Therefore Case 2 can be treated in the same way as Case 1. Using Proposition 4 we know the number and type of the singularities. For $0 \leq c<2$ the origin is a global center. For $c=2$ there is a cuspidal singularity at $(-1,0)$ and its direction is determined by the sign of $a+c$. For $c>2$ the cusp singularity splits into a saddle and center where the direction of the splitting is given by the sign of $a+c$. Using symmetry with respect to the $x$-axis and the sign of $\dot{\theta}$ we can complete the global phase portraits as in Case 1. Figure 6 summarizes the bifurcation diagram in the $(c, a)$-plane for $c \geq 0$ and $a+c \neq 0$. The bifurcation line $a+c=0$ in Figure 6 corresponds to Case 3 that was treated in section 3.

\subsection{Case 5: Three simple}

In this case the parameter $(a, b, c)$ satisfies $c \geq 0$ and $(a-2 b+c) b<0$. Furthermore we consider the necessary and sufficient condition

$$
(2 b-a) \sqrt{b /(3 b-a-c)}<2
$$

to ensure that all singularities appear to be collinear (see Proposition 2). Analogously to Case 1 we find that the global phase portraits are classified as in Figure 7. 


\section{Global phase portraits when $\mathcal{R}_{0}$ is triple}

In this section we study the global phase portraits in Case 4, i.e. of the vector fields $X_{(a, b, c)}^{R}$ with $c \geq 0, a=$ $2 b-c$ and $b \neq 0$. Then $A$ reads as

$$
A(\theta)=b \sin ^{3} \theta
$$

clearly $A$ only has a zero in $[0, \pi)$ at $\theta=0$ and its multiplicity is 3 .

First we study the local phase portrait along the ray $\mathcal{R}_{0}$ when it is triple; this study is summarized in Propositions 5, 6 and 7 and drawn in Figure 10. Next the global phase portrait is analyzed by using symmetry and the construction of a convenient positively invariant set.

\subsection{Local phase portrait along triple ray $\mathcal{R}_{0}$}

Proposition 5. Suppose that $c \geq 0,2 b-a-c=0$ and $a+c \neq 0$ (hence $b \neq 0)$.

(1) $B(\pi)=-c, A(\pi)=A^{\prime}(\pi)=A^{\prime \prime}(\pi)=0$ and $A^{\prime \prime \prime}(\pi)=-6 b \neq 0$.

(2) For $0 \leq c<2$ the only singularity is the center at the origin. It is a global center.

(3) For $c=2$ the vector field $X_{(a, b, c)}$ has two singularities, the center at $(0,0)$ and the non-elementary singularity at $(-1,0)$. The linearization at $(-1,0)$ is identically 0 and its topological type is established in Proposition 6.

(4) For $c>2$ the vector field $X_{(a, b, c)}$ has three singularities, the center at $(0,0)$ and nilpotent singularities $\left(-x_{ \pm}, 0\right)$ where $x_{ \pm}=x_{ \pm}(c)$ is defined in (11); their topological type is established in Proposition 7 and $0<x_{-}(c)<1<x_{+}(c)$.

Proof. By elimination of the parameter $a=2 b-c$ statement (1) follows directly from Proposition 3. In particular the vector field writes as

$$
\begin{aligned}
& \dot{x}=-y\left(1+c x+x^{2}+y^{2}\right), \\
& \dot{y}=x\left(1+c x+x^{2}+y^{2}\right)+b y^{2} .
\end{aligned}
$$

Clearly all singularities lie on the horizontal axis and those different from the origin satisfy $1+c x+x^{2}=0$ with $x \neq 0$. Notice that the Jacobian matrix at a singularity $(x, 0)$ for $x \neq 0$ equals

$$
J(x)=\left(\begin{array}{cr}
0 & 0 \\
x^{2}-1 & 0
\end{array}\right) .
$$

Now statements (2), (3) and (4) follow immediately.

If $0 \leq c<2$ then $X_{(2 b-c, b, c)}$ has a global center at $(0,0)$.

If $c=2$ then by Proposition 5 the vector field $X_{(2 b-c, b, c)}$ has two singularities at the negative horizontal axis: the center $(0,0)$ and a degenerate singularity $(-1,0)$. In Proposition 6 and Figure 10 the topological type of the singularity $(-1,0)$ is established depending on the sign of $b-1$.

If $c>2$ then by Proposition 5 the vector field $X_{(2 b-c, b, c)}$ has 3 singularities at the negative horizontal axis: the center $(0,0)$ and $\left(-x_{ \pm}, 0\right)$ where $x_{ \pm}$is defined in (11). In Proposition 7 and Figure 10 the type of the singularities $\left(-x_{ \pm}, 0\right)$ is established.

Proposition 6. Suppose that $2 b-a-c=0, a+c \neq 0$ (hence $b \neq 0)$ and $c=2$. Then the topological type of the non-elementary singularity of $X_{(a, b, 2)}$ at $(-1,0)$ depends on the sign of $b-1$, and the corresponding phase portrait along the ray $\mathcal{R}_{0}$ can be found in Figure 10 with $c=2, b \leq 1$ and $c=2, b>1$. More precisely,

(1) for $b \leq 1$ the orbits near the singularity at $(-1,0)$ form a 'flow box';

(2) for $b>1$ the singularity at $(-1,0)$ has one elliptic sector and three hyperbolic sectors.

Proof. From Proposition 5 we know that for $c=2$ there a unique singularity different from $(0,0)$ and it is situated at $(-1,0)$. Since $J(1)=0$ we have to perform a blow up to study this non-elementary singularity. 


$$
\begin{aligned}
& 0 \leq c<2 \\
& c=2, b \leq 1 \\
& c=2, b>1
\end{aligned}
$$

$c>2, b<0$

$c>2,0<b \leq b_{-}(c)$

$c>2, b_{-}(c)<b \leq b_{+}(c)$
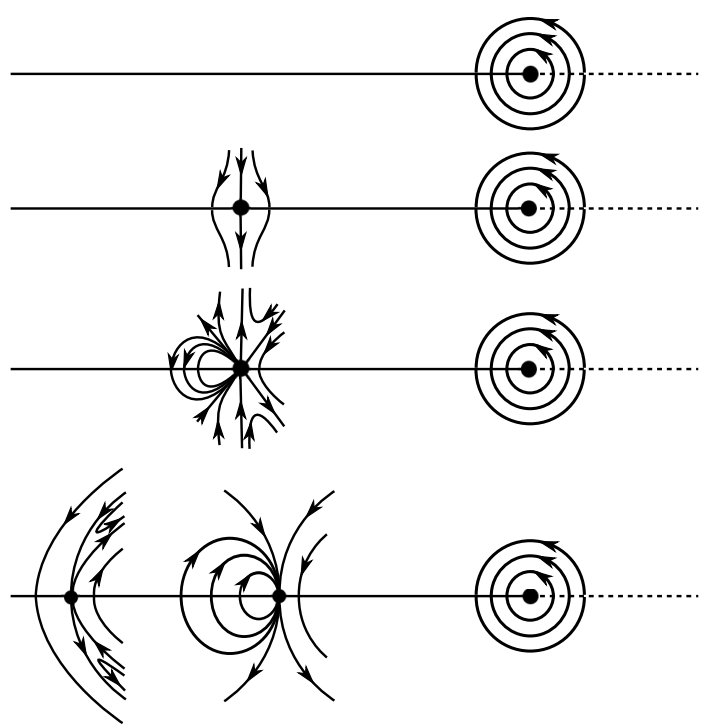

$$
c>2, b_{+}(c) \leq b
$$
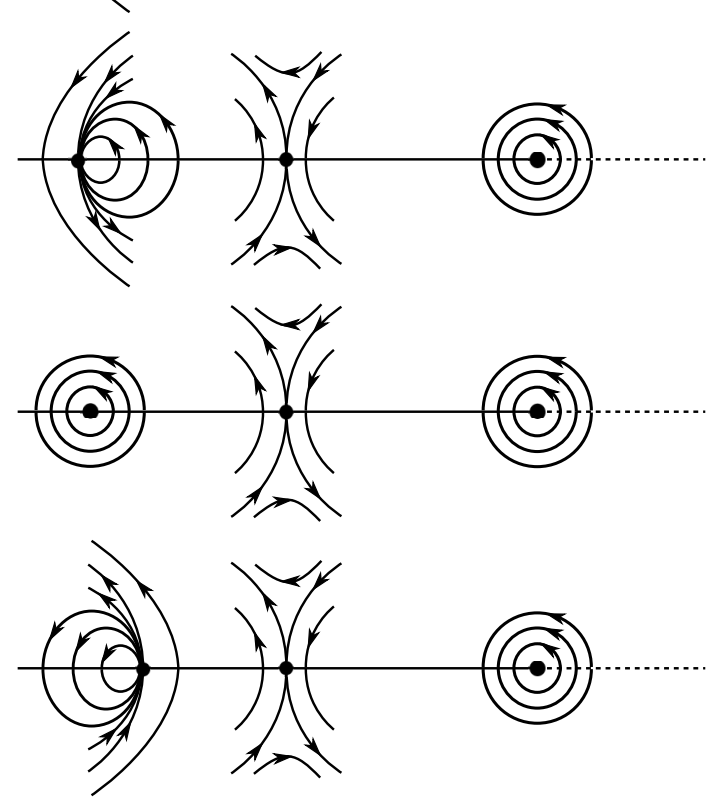

Fig. 10. Classification of the singularities on the horizontal axis in case $A^{\prime}(0)=A^{\prime \prime}(0)=0$ and $A^{\prime \prime \prime}(0) \neq 0$, see Propositions 5 , 6 and 7.

In case $c=2$ we use blow up to study the singularity and after blow up we obtain a finite number of singularities (hyperbolic, semi-hyperbolic and/or nilpotent depending on the sign of $b-1$ ); these singularities can be classified using Theorems 3 and 4 .

Suppose that $c=2$, then (15) in polar coordinates writes as

$$
\dot{r}=r^{2}\left(b \sin ^{2} \theta-1\right) \sin \theta, \dot{\theta}=r\left(b \sin ^{2} \theta-1\right) \cos \theta+r^{2} .
$$

System (16) when presented on the cylinder with radius 1 and coordinates $(r, \theta)$ has an invariant circle at $r=0$ full of singularities. For $r>0$ it is equivalent to

$$
\dot{r}=r\left(b \sin ^{2} \theta-1\right) \sin \theta \equiv R(r, \theta), \dot{\theta}=\left(b \sin ^{2} \theta-1\right) \cos \theta+r \equiv \Theta(r, \theta) .
$$

To study the phase portrait of (15) for $(x, y)=(r \cos \theta, r \sin \theta)$ with $r>0$ but arbitrary small we can study (17) for $r>0$. For $r>0$ the orbits of (16) correspond to the ones of (17) preserving direction of time; for $r<0$ the orbits of (16) correspond to the ones of (17) reversing direction of time. As for (16) the circle $\{r=0\}$ is invariant for (17) but now it consists only of a finite number of singularities: 2 hyperbolic singularities for $b<1,2$ nilpotent singularities for $b=1$ and 6 elementary $(2$ hyperbolic and 4 
semi-hyperbolic) singularities for $b>1$. The direction of the regular orbits on the circle $\{r=0\}$ is given by the sign of $\Theta(0, \theta)$.

We now study the topological type of the singularities of (17) on the circle $\{r=0\}$. Since $R(r, 2 \pi-\theta)=-R(r, \theta)$ and $\Theta(r, 2 \pi-\theta)=\Theta(r, \theta)$ (resp. $R(-r, \pi-\theta)=R(r, \theta)$ and $\Theta(-r, \pi-\theta)=$ $\Theta(r, \theta))$ system (17) is invariant under the transformation $\bar{T}_{1}(r, \theta, t)=(r, 2 \pi-\theta,-t)$ (resp. $\bar{T}_{2}(r, \theta, t)=$ $(-r, \pi-\theta, t))$. Therefore if $\left(0, \theta_{0}\right)$ is a singularity of $(17)$, then $\left(0, k \pi-\theta_{0}\right)$ for $k=1,2$ and $\left(0, \pi+\theta_{0}\right)$ are singularities of (17); in particular their nearby local phase portraits can be deduced from the one near $\left(0, \theta_{0}\right)$ by use of the appropriate transformations $\bar{T}_{1}$ and $\bar{T}_{2}$.

For $b \leq 1$ system (17) has 2 singularities on the circle $\{r=0\}$ situated at $\theta_{0}=\pi / 2$ and $\theta_{0}+\pi$. The Jacobian matrix at $(0, \pi / 2)$ is given by

$$
\left(\begin{array}{cc}
b-1 & 0 \\
1 & -(b-1)
\end{array}\right)
$$

If $b<1$ then the singularities are hyperbolic saddle points; the stable and unstable manifold at $(0, \pi / 2)$ are tangent to the $r$ - and $\theta$-direction respectively while the stable and unstable manifolds at $(0,3 \pi / 2)$ are tangent to the $\theta$ - and $r$-direction resp. by the invariance under $\bar{T}_{2}$.

If $b=1$ then the 2 singularities on the circle $\{r=0\}$ are nilpotent. By transformation $(u, v)=$ $(\theta-\pi / 2, r)$ system (15) is brought in normal form (14); the functions $\mathcal{C}, F$ and $G$ that appear in Theorem 4 here are given, when $u \rightarrow 0$, by

$$
\mathcal{C}(u)=-\sin ^{3} u, F(u)=\sin ^{5} u \cos u=u^{5}+O\left(u^{7}\right) \text { and } G(u)=2 \sin ^{2} u \cos u=2 u^{2}+O\left(u^{4}\right) .
$$

Therefore by Theorem 4 the singularity at $(0, \pi / 2)$ is a saddle for which the stable (resp. unstable) manifold is tangent to the $\theta$-axis (resp. $r$-axis) at $(r, \theta)=(0, \pi / 2)$. By symmetry we obtain the phase portrait near the singularity at $(0,3 \pi / 2)$ from $(0, \pi / 2)$ after reversing time. The blow up procedure for $b \leq 1$ is shown in Figure 12.

If $b>1$ then (17) has 6 singularities on the circle $\{r=0\}$ situated at

$$
\theta_{0} \equiv \pi / 2, \theta_{1} \equiv \arcsin \frac{1}{\sqrt{b}}, \pi-\theta_{1}, \pi+\theta_{1}, \theta_{0}+\pi \text { and } 2 \pi-\theta_{1} .
$$

The Jacobian matrix at $\theta_{0}$ and $\theta_{0}+\pi$ equals the one in (18); therefore these singularities are hyperbolic saddle points as for $b<1$ but the stable and unstable manifolds are reversed. The singularity $\theta_{1}$ is semihyperbolic and its Jacobian matrix is given by

$$
\left(\begin{array}{ll}
0 & 0 \\
1 & \mu
\end{array}\right) \text { with } \mu=\frac{2(b-1)}{\sqrt{b}} .
$$

After transformation $(u, v)=\left(r, \theta-\theta_{1}+r / \mu\right)$ the system (17) is written in normal form (12) to study the semi-hyperbolic singularity $\left(0, \theta_{1}\right)$ of $(17)$ with

$$
\begin{aligned}
& f(u, v)=R\left(u, v+\theta_{1}-u / \mu\right), \\
& g(u, v)=\Theta\left(u, v+\theta_{1}-u / \mu\right)+\frac{R\left(u, v+\theta_{1}-u / \mu\right)}{\mu}-\mu v .
\end{aligned}
$$

The asymptotics of the curve $v=\mathcal{C}(u)$ for $u \rightarrow 0$ along which $g(u, v)+\mu v=0$ is given by

$$
\mathcal{C}(u)=-\frac{b \sqrt{b-1}(b-4)}{8} u^{2}+O\left(u^{3}\right) \text { for } b \neq 4 \text { and } \mathcal{C}(u)=-\frac{1}{18} u^{3}+O\left(u^{4}\right) \text { for } b=4 .
$$

For all $b>1$ the behavior of the flow of $\dot{u}=f(u, v)$ on $v=\mathcal{C}(u)$ is given by

$$
\dot{u}=F(u) \equiv f(u, \mathcal{C}(u))=-\frac{1}{\sqrt{b-1}} u^{2}+O\left(u^{3}\right), u \rightarrow 0 .
$$

By Theorem 3 it follows that the singularity $\left(0, \theta_{1}\right)$ is a saddle-node with 2 hyperbolic sectors for $r>0$ and a parabolic sector for $r<0$. It has an unstable manifold tangent to $\left\{\theta=\theta_{1}\right\}$ at $\left(0, \theta_{1}\right)$ and center manifolds tangent to $r=0$ and infinitely mutually tangent at $\left(0, \theta_{1}\right)$. In Figure 11 the local phase portraits are thus drawn near $\left(0, \theta_{1}\right),\left(0, \pi-\theta_{1}\right),\left(0, \pi+\theta_{1}\right)$ and $\left(0,2 \pi-\theta_{1}\right)$ using the adequate transformations $\bar{T}_{1}$ and $\bar{T}_{2}$. 

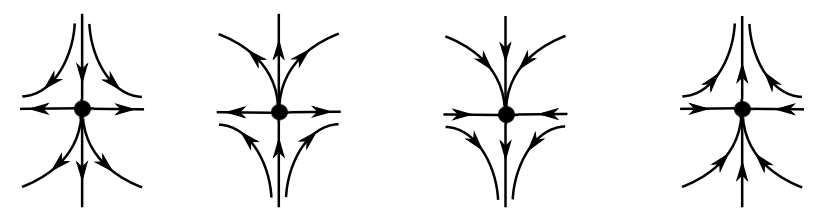

$\theta=\theta_{1}$

$$
\theta=\pi-\theta_{1} \quad \theta=\pi+\theta_{1} \quad \theta=2 \pi-\theta_{1}
$$

Fig. 11. Local phase portraits in polar coordinates $(\theta, r)$ near $\left(\theta_{1}, 0\right),\left(\pi-\theta_{1}, 0\right),\left(\pi+\theta_{1}, 0\right)$ and $\left(2 \pi-\theta_{1}, 0\right)$ using the transformations $\bar{T}_{1}$ and $\bar{T}_{2}$, see proof of Proposition 6 and (19) for the definition of $\theta_{1}$.

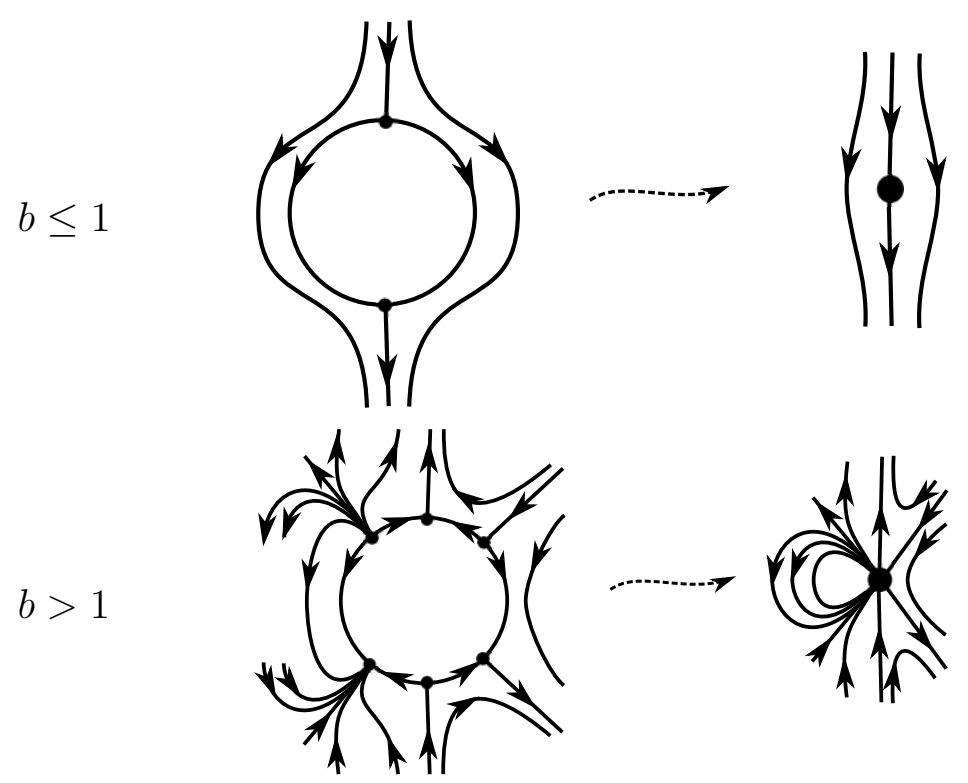

Fig. 12. Blow-up procedure at $(-1,0)$, see proof of Proposition 6 .

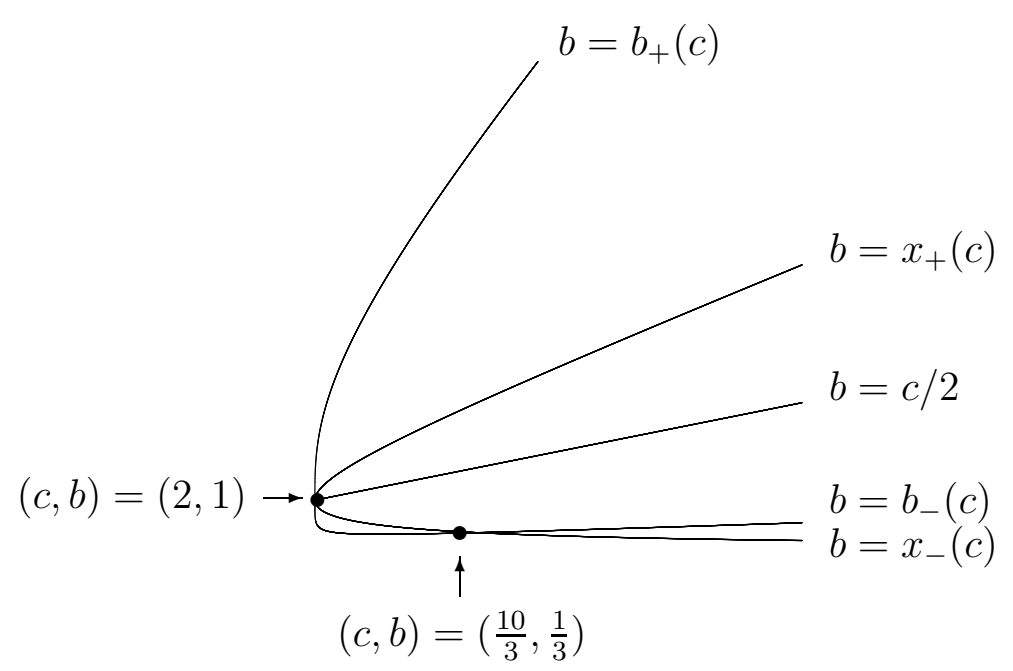

Fig. 13. Bifurcation lines in the plane $a=2 b-c$, see Propositions 5 and 7 .

The blow up procedure that determines the topological type of the singularity $(-1,0)$ of $(15)$ for $b>1$ is summarized in Figure 12.

Proposition 7. Suppose that $2 b-a-c=0, a+c \neq 0$ (hence $b \neq 0$ ) and $c>2$. Then $X_{(a, b, c)}$ has two 
nilpotent singularities $\left(-x_{ \pm}, 0\right)$ where $x_{ \pm}$is defined by (11). Consider the functions $b_{ \pm}$defined by

$$
b_{ \pm}(c)=\frac{c}{2}+\sqrt{c^{2}-4} \pm \sqrt{c^{2}-4+c \sqrt{c^{2}-4}} .
$$

Then the nearby phase portrait can be classified as (see Figure 10):

(1) If $b<0$ then the nilpotent singularity $\mathbf{s}_{-}$has one hyperbolic and one elliptic sector and $\mathbf{s}_{+}$is a nilpotent saddle.

(2) If $0<b \leq b_{-}(c)$ then $\mathbf{s}_{-}$is a nilpotent saddle and the nilpotent singularity $\mathbf{s}_{+}$has one hyperbolic and one elliptic sector.

(3) If $b_{-}(c)<b<b_{+}(c)$ then $\mathbf{s}_{-}$is a nilpotent saddle and $\mathbf{s}_{+}$is a nilpotent center.

(4) If $b \geq b_{+}(c)$ then $\mathbf{s}_{-}$is a nilpotent saddle and the nilpotent singularity $\mathbf{s}_{+}$has one hyperbolic and one elliptic sector.

(5) Let $(b, c)$ be such that $c>2, b \neq 0$ and $b \notin\left(b_{-}(c), b_{+}(c)\right)$ and $\mathbf{s}_{ \pm}=\left(x_{0}, 0\right)$ the corresponding singularities. Then their separatrices can locally be written as the graph

$$
x=\mathcal{H}_{i}\left(y^{2}, \mathbf{s}_{ \pm}\right)=x_{0}+\kappa_{i}\left(\mathbf{s}_{ \pm}\right) y^{2}+O\left(y^{4}\right) \text { for } y \rightarrow 0, i=1,2,
$$

for a smooth function $Y \mapsto \mathcal{H}_{i}\left(Y, \mathbf{s}_{ \pm}\right), i=1,2$ that is not necessarily unique. The first non-zero coefficient $\kappa_{i}\left(\mathbf{s}_{ \pm}\right), i=1,2$ in the expansion equal

$$
\kappa_{i \pm} \equiv \kappa_{i}\left(\mathbf{s}_{ \pm}\right)= \pm \frac{1}{2} \frac{(c-2 b) \pm 2 \sqrt{c^{2}-4}+(-1)^{i+1} \sqrt{(c-2 b)^{2} \mp 8 b \sqrt{c^{2}-4}}}{c \sqrt{c^{2}-4} \pm\left(c^{2}-4\right)} ;
$$

the relative order and sign of $\kappa_{i \pm}, i=1,2$ can be found in (25) and (26).

(6) In particular as sketched in Figure 13 the curves $b_{ \pm}$and $x_{ \pm}$have the following relations and asymptotics:

(a) $0<b_{-}(c)<c / 2<x_{+}(c)<b_{+}$(c) for $c<2$,

(b) $b_{-}(c)=x_{-}(c)$ for $c=2$ or $c=10 / 3$,

(c) $b_{-}(c)<x_{-}(c)<c / 2$ for $2<c<10 / 3$ and $0<x_{-}(c)<b_{-}(c)$ for $c>10 / 3$,

(d) $\lim _{c \downarrow 2} b_{ \pm}(c)=\lim _{c \downarrow 2} x_{ \pm}(c)=1$ and $\lim _{c \rightarrow+\infty} b_{ \pm}(c)=\lim _{c \rightarrow+\infty} x_{ \pm}(c)=+\infty$.

Proof. From Proposition 5 we know that for $c>2$ there are two nilpotent singularities different from $(0,0)$ and they are situated at $\mathbf{s}_{ \pm}=\left(-x_{ \pm}, 0\right)$. All elements of the Jacobian matrix at $\mathbf{s}_{ \pm}, J\left(x_{ \pm}\right)$, vanish except the element $J\left(x_{ \pm}\right)_{2,1}=\left(c^{2}-4 \pm c \sqrt{c^{2}-4}\right) / 2 \equiv 1 /\left(2 \mu_{ \pm}\right)$. Since $0<c-\sqrt{c^{2}-4}<2<-c+\sqrt{c^{2}-4}$ for $c>2$ (hence $\operatorname{sgn}\left(\mu_{ \pm}\right)= \pm 1$ ) the singularities $\mathbf{s}_{ \pm}$are nilpotent. First by using Theorem 4 we will classify its topological type. Next we derive the relative position and the contact of the separatrices at $\mathbf{s}_{ \pm}$.

By transformation $(u, v)=\left(y, x+x_{ \pm}\right)$and time rescaling $\tau=\left(2 \mu_{ \pm}\right)^{-1} t$ the vector field $X_{(a, b, c)}$ is transformed into (14) to study the topological type of $\mathbf{s}_{ \pm}$by Theorem 4 ; the asymptotics of the functions $F_{ \pm}, G_{ \pm}$and $\mathcal{C}_{ \pm}$for $u \rightarrow 0$ is given by

$$
\begin{aligned}
& F_{ \pm}(u)=\alpha_{ \pm} u^{3}+O\left(u^{4}\right) \text { with } \alpha_{ \pm} \equiv \mp 4 \mu_{ \pm}^{2} b \sqrt{c^{2}-4} \\
& G_{ \pm}(u)=\beta_{ \pm} u \text { with } \beta_{ \pm}=2(2 b-c) \mu_{ \pm} \\
& \mathcal{C}_{ \pm}(u)=\kappa_{ \pm} u^{2}+O\left(u^{3}\right) \text { with } \kappa_{ \pm}=\mp\left(c-2 b \pm \sqrt{c^{2}-4}\right) \mu_{ \pm} \\
& \delta_{ \pm}=\left(\beta_{ \pm}^{2}+8 \alpha_{ \pm}\right)=\mu_{ \pm}^{2}\left[(2 b-c)^{2} \mp 8 b \sqrt{c^{2}-4}\right] .
\end{aligned}
$$

Notice that for all $b \neq 0$ we have $\delta_{-}>0$ and we can write

$$
\delta_{+}=4 \mu_{+}^{2}\left(b-b_{-}(c)\right)\left(b-b_{+}(c)\right),
$$

where $b_{ \pm}$is defined in (20). In particular $\delta_{+} \geq 0$ for $b \leq b_{-}(c)$ or $b \geq b_{+}(c)$ and $\delta_{+}<0$ for $b_{-}(c)<b<$ $b_{+}(c)$.

For $b>0$ we encounter two singular cases, the Hamiltonian triple reversible case $c=2 b$ and the case $b=x_{ \pm}(c)$. 
Let us first suppose that $b>0, c \neq 2 b$ and $b \neq x_{ \pm}(c)$. Since then $\alpha_{-}>0$ the singularity $\mathbf{s}_{-}$is a saddle for $b>0$. On the other hand then $\alpha_{+}<0$ and so the sign of $\delta_{+}$determines the topological behavior of $\mathbf{s}_{+}$. Therefore for $b \leq b_{-}(c)$ or $b \geq b_{+}(c)$ the phase portrait at the singularity $\mathbf{s}_{+}$exists of one hyperbolic and one elliptic sector and for $b_{-}(c)<b<b_{+}(c)$ the singularity $\mathbf{s}_{+}$is a center (a focus is not possible since $\mathbf{s}_{+}$ lies on the line of symmetry).

In case $c=2 b$ then $G(u) \equiv 0$ and $\operatorname{sgn}\left(\alpha_{ \pm}\right)=\mp 1$ and therefore $\mathbf{s}_{+}$is a center and $\mathbf{s}_{-}$is a (nonlinear) saddle which is in agreement with the general case for $b>0$ and $b_{-}(c)<b<b_{+}(c)$. In case $b=x_{ \pm}(c)$ the curve $\mathcal{C}_{ \pm}(u) \equiv 0$ is an isocline such that $\dot{u}=0$ but the sign of $\alpha_{ \pm}$does not change. Therefore $\mathbf{s}_{-}$is a saddle and since $b_{-}(c)<x_{+}(c)<b_{+}(b)$ the quantity $\delta_{+}<0$ implying that $\mathbf{s}_{+}$is a center. Remark that passing through the bifurcation curve $b=x_{-}(c)$ the order of contact of the separatrices at $\mathbf{s}_{-}$changes (increases) but the topological type of $\mathbf{s}_{-}$remains unchanged.

For $b<0$ one has $\alpha_{+}>0$ and hence $\mathbf{s}_{+}$is a saddle. On the other hand $\alpha_{-}<0$ and $\delta_{-}>0$, therefore $\mathbf{s}_{-}$is a nilpotent singularity with one hyperbolic and one elliptic sector.

Let $(b, c)$ be an arbitrary parameter with $c>2, b \neq 0$ and $b \notin\left(b_{-}(c), b_{+}(c)\right)$ and $\mathbf{s}_{ \pm}=\left(x_{0}, 0\right)$ the corresponding singularities. Then their separatrices can locally be written as in (21) where $\kappa_{i}\left(\mathbf{s}_{ \pm}\right), i=1,2$ is determined by the invariance property

$$
\dot{x}=\left(2 \kappa_{i}\left(\mathbf{s}_{ \pm}\right) y+O\left(y^{3}\right)\right) \dot{y} \text { along the separatrix } x=\mathcal{H}_{i}\left(y^{2}, \mathbf{s}_{ \pm}\right) .
$$

By substitution of the asymptotic expansion in (21) and identifying equal powers of $y$ one finds

$$
\kappa_{i}\left(\mathbf{s}_{ \pm}\right) \equiv \frac{1}{4} \frac{4 x_{0}+c+2 b+(-1)^{i} \sqrt{16 x_{0} b+(c+2 b)^{2}}}{1-x_{0}^{2}} \text { for } i=1,2,
$$

and correspondingly the asymptotic behavior along the separatrices for $y \rightarrow 0$

$$
\dot{y}=\zeta_{i}\left(\mathbf{s}_{ \pm}\right) y^{2}+O\left(y^{3}\right) \text { along the separatrix } x=\mathcal{H}_{i}\left(y^{2}, \mathbf{s}_{ \pm}\right),
$$

where

$$
\zeta_{i}\left(\mathbf{s}_{ \pm}\right) \equiv b+x_{0}-\left(2+c x_{0}\right) \kappa_{i}\left(\mathbf{s}_{ \pm}\right) .
$$

Substituting $\mathbf{s}_{ \pm}=\left(\left(-c \pm \sqrt{c^{2}-4}\right) / 2,0\right)$ in (23) and (24) we obtain (22) and

$$
\zeta_{i \pm} \equiv \zeta_{i}\left(s_{ \pm}\right)=-\frac{1}{4}\left[(c-2 b)+(-1)^{i} \sqrt{(c-2 b)^{2} \mp 8 b \sqrt{c^{2}-4}}\right] \text { for } i=1,2 .
$$

It can be checked that all quantities $\kappa_{i}\left(\mathbf{s}_{ \pm}\right)$and $\zeta_{i}\left(\mathbf{s}_{ \pm}\right)$are different from 0 and $\kappa_{1}\left(\mathbf{s}_{ \pm}\right)=\kappa_{2}\left(\mathbf{s}_{ \pm}\right)$if and only if $b=b_{ \pm}(c)$. Furthermore

\begin{tabular}{c|l} 
& $\mathbf{s}_{+}$ \\
\hline$b<0$ & $0<\kappa_{2+}<\kappa_{1+}$ and $\zeta_{2+}<0<\zeta_{1+}$ \\
$0<b \leq b_{-}(c)$ & $0<\kappa_{2+} \leq \kappa_{1+}$ and $\zeta_{2+} \leq \zeta_{1+}<0$ \\
$b_{+}(c) \leq b$ & $\kappa_{2+} \leq \kappa_{1+}<0$ and $0<\zeta_{2+} \leq \zeta_{1+}$
\end{tabular}

and

$$
\begin{array}{c|c} 
& \mathbf{s}_{-} \\
\hline b<0 & \kappa_{1-}<0<\kappa_{2-} \text { and } \zeta_{2-}<\zeta_{1-}<0 \\
b>0 & \kappa_{1-}<0<\kappa_{2-} \text { and } \zeta_{2-}<0<\zeta_{1-}
\end{array}
$$

summarize all the possible situations.

It follows that for $b>0$ the elliptic sector at $\mathbf{s}_{+}$is determined by $\left\{x=\mathcal{H}_{i}\left(y^{2}, \mathbf{s}_{+}\right)\right\}$and the hyperbolic sector at $\mathbf{s}_{+}$is determined by $\left\{x=\mathcal{H}_{j}\left(y^{2}, \mathbf{s}_{+}\right)\right\}$with $(i, j)=(1,2)$ for $0<b \leq b_{-}(c)$ and with $(i, j)=(2,1)$ for $b \geq b_{+}(c)$; for $b<0$ the elliptic sector at $\mathbf{s}_{-}$is determined by $\left\{x=\mathcal{H}_{1}\left(y^{2}, \mathbf{s}_{-}\right)\right\}$and the hyperbolic sector at $\mathbf{s}_{-}$is determined by $\left\{x=\mathcal{H}_{2}\left(y^{2}, \mathbf{s}_{-}\right)\right\}$.

Notice that all trajectories in the elliptic sector at $\mathbf{s}_{ \pm}$make contact of infinite order at $\mathbf{s}_{ \pm}$but there is only one that is analytic. 


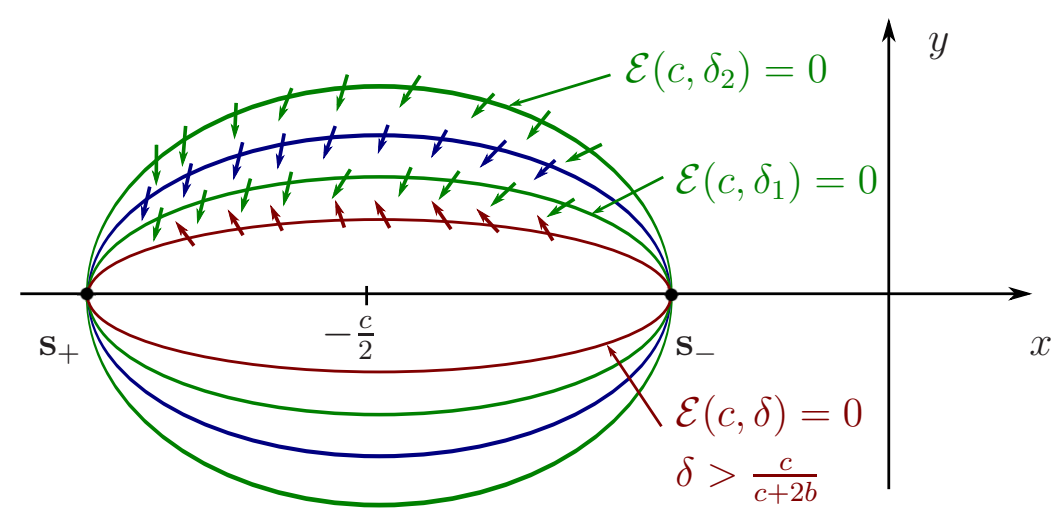

Fig. 14. Trapping regions see Lemma 3.

The stable and unstable manifolds at the saddles $\mathbf{s}_{ \pm}$for $\operatorname{sgn}(b)=\mp 1$, say $\mathcal{V}\left(\mathbf{s}_{ \pm}\right)$and $\mathcal{U}\left(\mathbf{s}_{ \pm}\right)$, are given by

$$
\begin{aligned}
\mathcal{V}\left(\mathbf{s}_{ \pm}\right) & =\left\{x=\mathcal{H}_{1}\left(y^{2}, \mathbf{s}_{ \pm}\right), y \leq 0\right\} \cup\left\{x=\mathcal{H}_{2}\left(y^{2}, \mathbf{s}_{ \pm}\right), y \geq 0\right\} \\
\mathcal{U}\left(\mathbf{s}_{ \pm}\right) & =\left\{x=\mathcal{H}_{1}\left(y^{2}, \mathbf{s}_{ \pm}\right), y \leq 0\right\} \cup\left\{x=\mathcal{H}_{2}\left(y^{2}, \mathbf{s}_{ \pm}\right), y \leq 0\right\}
\end{aligned}
$$

The local phase portraits near the singularities are drawn in Figure 10 taking into account the asymptotic behavior of the separatrices.

\subsection{Global phase portrait}

The global phase portraits drawn in Figure 15 are rather easily obtained using the argument of symmetry except when $c>2$ and $0<b \leq b_{-}(c)$. To complete the global phase portrait in case $c>2$ and $0<b \leq$ $b_{-}(c)$ we need to know whether there is a trajectory connecting $\mathbf{s}_{+}$and $\mathbf{s}_{-}$(see Proposition 8 ). Therefore in Lemma 3 we will construct a positively invariant region, also called trapping region. Roughly speaking the following is meant by the notion 'trapping region' $\mathcal{T}$ : if a trajectory starts in $\mathcal{T}$, then it is forced to remain in $\mathcal{T}$ or in other words it is trapped within it.

Lemma 3. Let $c>2$ and $0<b \leq b_{-}(c)$ arbitrary but fixed where $b_{-}$is defined in (20). For $\delta>0$ we define the ellipse $\mathcal{E} \equiv \mathcal{E}(c, \delta)=0$ with center at $(-c / 2,0)$ and $\sqrt{c^{2}-4}$ resp. $\sqrt{c^{2}-4} / \delta$ the horizontal resp. vertical semi-axes (see Figure 14), i.e.

$$
\mathcal{E}(x, y) \equiv 1+c x+x^{2}+\delta y^{2} .
$$

Let $\dot{\mathcal{E}}$ be the directional derivative of $\mathcal{E}$ with respect to the vector field $X_{(b, c)}$ where $X_{(b, c)} \equiv X_{(2 b-c, b, c)}^{R}$ and $X^{R}$ is defined in $(2)$. Then $\left.\dot{\mathcal{E}}\right|_{\mathcal{E}=0, y>0}>0$ for all $\delta>c /(2 b+c)$ and there exist $0<\delta_{2}=\delta_{2}(b, c) \leq \delta_{1}=$ $\delta_{1}(b, c)<1$ such that $\left.\dot{\mathcal{E}}\right|_{\mathcal{E}=0, y>0}<0$ for all $\delta_{2} \leq \delta \leq \delta_{1}$. In particular

$$
\delta_{i}=2 \sqrt{c^{2}-4} \kappa_{i+}
$$

where $\kappa_{i+}$ is defined in (22) for $i=1,2$. Furthermore $\delta_{1}(b, c)=\delta_{2}(b, c)$ if and only $b=b_{-}(c)$.

Proof. By definition we have $\dot{\mathcal{E}}=(c+2 x) \dot{x}+2 \delta y \dot{y}$ and therefore on $\mathcal{E}(x, y)=0$ this identity reduces for $|x+c / 2|<\sqrt{c^{2}-4} / 2$ to

$$
\left.\dot{\mathcal{E}}\right|_{\mathcal{E}(x, y)=0}=-y^{3}\left[2(\delta-1)^{2} x+c(1-\delta)-2 \delta b\right] \equiv-y^{3} P(x, \delta, b, c) .
$$

Clearly for $\delta>c(2 b+c)$ the factor $P(x, \delta, b, c)$ is negative and therefore $\left.\dot{\mathcal{E}}\right|_{\mathcal{E}=0, y>0}>0$ for all $\delta>$ $c /(2 b+c)$. Therefore necessarily $\delta_{1}<1$ if such $\delta_{1}$ as described in the statement exists. 
The factor $P(x, \delta, b, c)$ has a sign change at $x=x_{*}$ with

$$
x_{*} \equiv x_{*}(b, c, \delta)=\frac{c(\delta-1)+2 \delta b}{2(\delta-1)^{2}} ;
$$

more precisely $P(x, \delta, b, c)>0$ for $x>x_{*}$ and $P(x, \delta, b, c)<0$ for $x<x_{*}$. To complete the proof of the proposition it suffices to show that there exists a range $\delta_{2}(b, c) \leq \delta_{1}(b, c)$ such that for all $\delta_{2}(b, c) \leq \delta \leq$ $\delta_{1}(b, c)$ the vertical line $x=x_{*}$ lies on the left of the ellipse, or equivalently

$$
0>2 x_{*}(b, c, \delta)+c+\sqrt{c^{2}-4} \equiv \frac{Q(b, c, \delta)}{(\delta-1)^{2}} .
$$

The map $Q(b, c, \delta)$ defined by $(27)$ is a quadratic polynomial in $\delta$ whose coefficients are smooth functions in $(b, c)$ :

$$
Q(b, c, \delta)=\left(\sqrt{c^{2}-4}+c\right) \delta^{2}+\left(2 b-c-2 \sqrt{c^{2}-4}\right) \delta+\sqrt{c^{2}-4}
$$

For $0<b \leq b_{-}(c), c>2$ the coefficients are consecutively positive, negative and positive; therefore by Descartes' rule $Q(b, c, \cdot)$ has no negative roots $\delta \leq 0$. On the other hand the discriminant $\Delta(b, c)$ of $Q(b, c, \cdot)$ is strictly positive for $0<b<b_{-}(c), c>2$. Therefore there exist $0<\delta_{2}=\delta_{2}(b, c) \leq \delta_{1}=\delta_{1}(b, c)$ with $Q\left(b, c, \delta_{i}\right)=0$ for $i=1,2, Q(b, c, \delta)<0$ for all $\delta<\delta_{2}$ and $Q(b, c, \delta)>0$ for all $\delta_{2}<\delta<\delta_{1}$. Applying the discriminant rule for the roots of the quadratic polynomial (28) in $\delta$ we can write down explicit expressions for $\delta_{i}, i=1,2$ :

$$
\delta_{i}(b, c)=\frac{1}{2} \frac{c-2 b+2 \sqrt{c^{2}-4}+(-1)^{i} \sqrt{(c-2 b)^{2}-8 b \sqrt{c^{2}-4}}}{c+\sqrt{c^{2}-4}} .
$$

By the definition of $b_{-}$it follows that $\delta_{1}=\delta_{2}$ if and only if $b=b_{-}(c)$. Comparing both formulas (22) and (29) one finds easily the relation between $\delta_{i}(b, c)$ and $\kappa_{i+}, i=1,2$.

Proposition 8. Let $c>2,0<b \leq b_{-}(c)$ and $a=c-2 b$. Consider the separatrices $\left\{x=\mathcal{H}_{i}\left(y^{2}, \mathbf{s}_{ \pm}\right)\right\}, i=$ 1,2 at the nilpotent singularity $\mathbf{s}_{+}$and the saddle $\mathbf{s}_{-}$in the phase portrait of $X_{(a, b, c)}$ as in $(21)$. Then there is exactly one trajectory in the family $\left\{x=\mathcal{H}_{1}\left(y^{2}, \mathbf{s}_{+}\right), y \leq 0\right\}$ (resp. $\left\{x=\mathcal{H}_{1}\left(y^{2}, \mathbf{s}_{+}\right), y \geq 0\right\}$ ) at $\mathbf{s}_{+}$that forms part of the stable manifold $\mathcal{V}^{-}\left(\mathbf{s}_{-}\right) \equiv\left\{x=\mathcal{H}_{1}\left(y^{2}, \mathbf{s}_{-}\right), y \leq 0\right\}$ (resp. unstable manifold $\mathcal{U}^{+}\left(\mathbf{s}_{-}\right) \equiv$ $\left.\left\{x=\mathcal{H}_{1}\left(y^{2}, \mathbf{s}_{-}\right), y \geq 0\right\}\right)$ at $\mathbf{s}_{-}$thus forming a '2-cycle'. Furthermore the phase portrait is sketched in Figure 16 .

Proof. Let $c>2,0<b \leq b_{-}(c)$ arbitrary but fixed and let $\delta_{1}=\delta_{1}(b, c)$ as defined in (29). Clearly by symmetry with respect to the $x$-axis the stable manifold at $\mathbf{s}_{-}$in the upper half plane $\{y>0\}$ and the unstable manifold at $\mathbf{s}_{-}$in the lower half plane $\{y<0\}$ connect enclosing the center at the origin.

By symmetry with respect to the $x$-axis and Bendixson's theorem there are only two possibilities left to finish the global phase portrait. In one case the stable manifold at $\mathbf{s}_{-}$in the lower half plane $\{y<0\}$ and the unstable manifold at $\mathbf{s}_{-}$in the upper half plane $\{y>0\}$ connect forming a double homoclinic loop (figure-eight) enclosing the nilpotent singularity $\mathbf{s}_{+}$by the left homoclinic loop and enclosing the center in the right homoclinic loop. In the other case the stable manifold at $\mathbf{s}_{-}$in the lower half plane $\{y<0\}$ and the unstable manifold at $\mathbf{s}_{-}$in the upper half plane $\{y>0\}$ connect at $\mathbf{s}_{+}$forming a figure-eight of which the right loop is the homoclinic loop enclosing the center at the origin and the left loop is made of a elliptic sector at $\mathbf{s}_{+}$bounded by heteroclinic connections between $\mathbf{s}_{+}$and $\mathbf{s}_{-}$. In both cases the figure-eight is encircled by a period annulus extending to infinity.

If we can prove that there exists a connection between the singularities $\mathbf{s}_{+}$and $\mathbf{s}_{-}$then the first case is excluded and we are done.

By symmetry of the vector field $X_{(a, b, c)}$ with respect to the $x$-axis it suffices to prove the statement concerning the connection in the upper half plane $\{y \geq 0\}$ only. To prove this we use Lemma 3 to construct a convenient trapping region $\mathcal{T}_{\delta_{0}}$. 


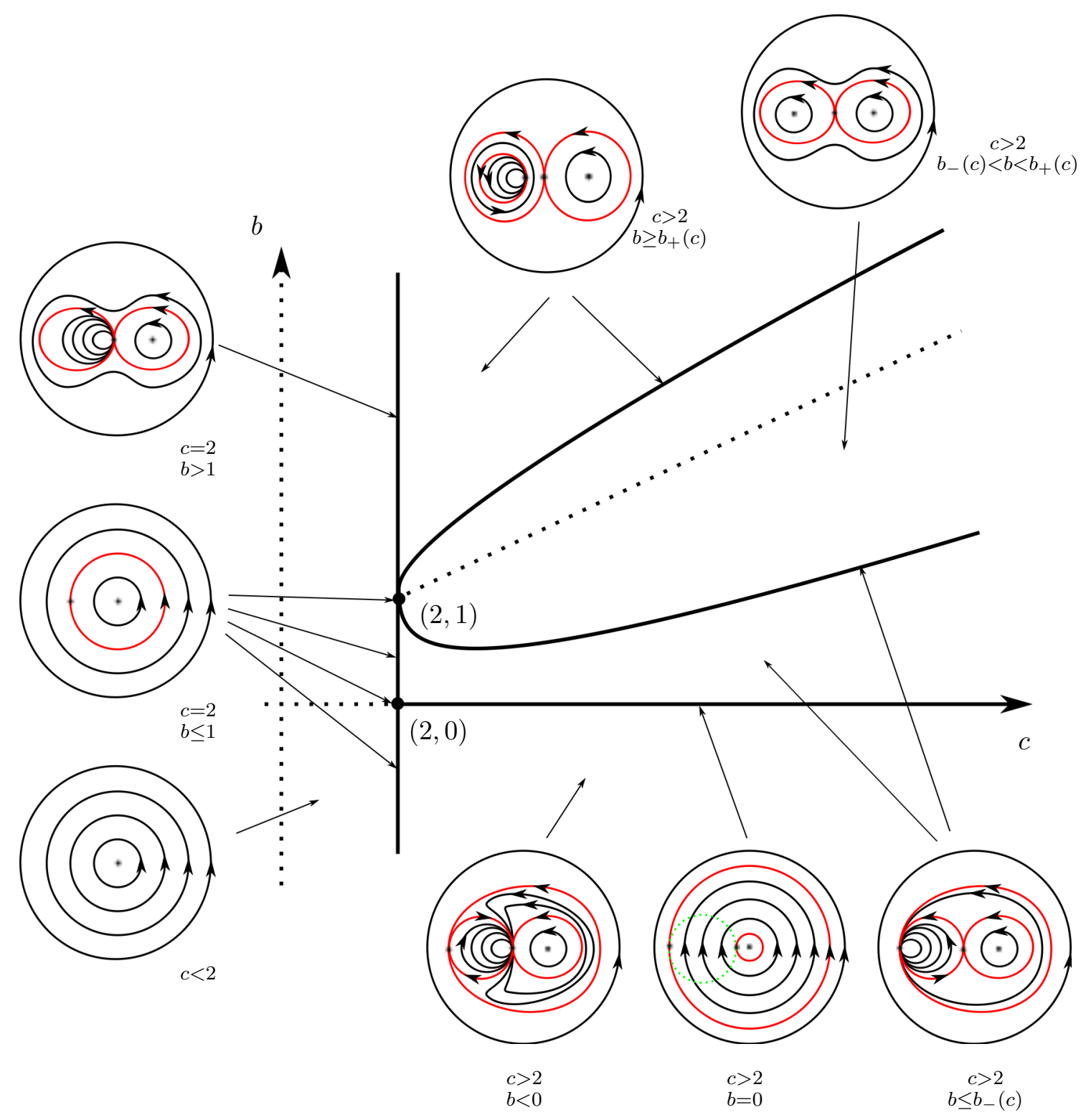

Fig. 15. Global phase portraits of $X_{(a, b, c)}^{R}$ for $a=2 b-c, c \geq 0$.

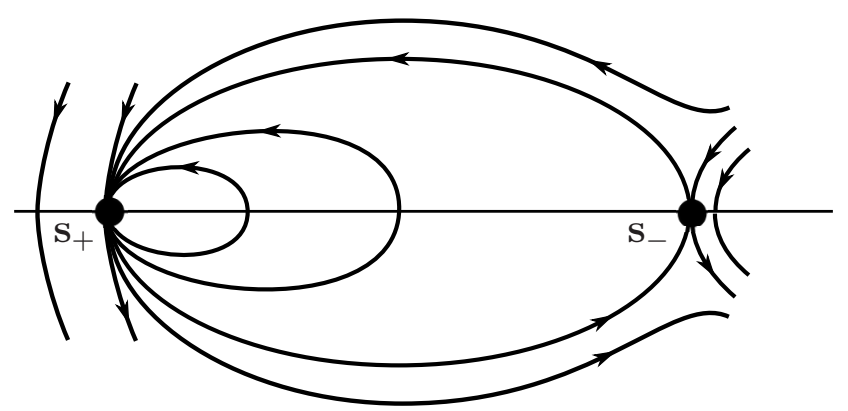

Fig. 16. Connections between the singularities $\mathbf{s}_{+}$and $\mathbf{s}_{-}$, see Proposition 8 .

For $\delta_{0}>1$ we define $\mathcal{T}_{\delta_{0}}$ by the ellipsoidal upper half disc

$$
\mathcal{B}_{\delta_{0}}^{*} \equiv\left\{1+c x+x^{2}+\delta_{0} y^{2} \leq 0, y \geq 0\right\}
$$




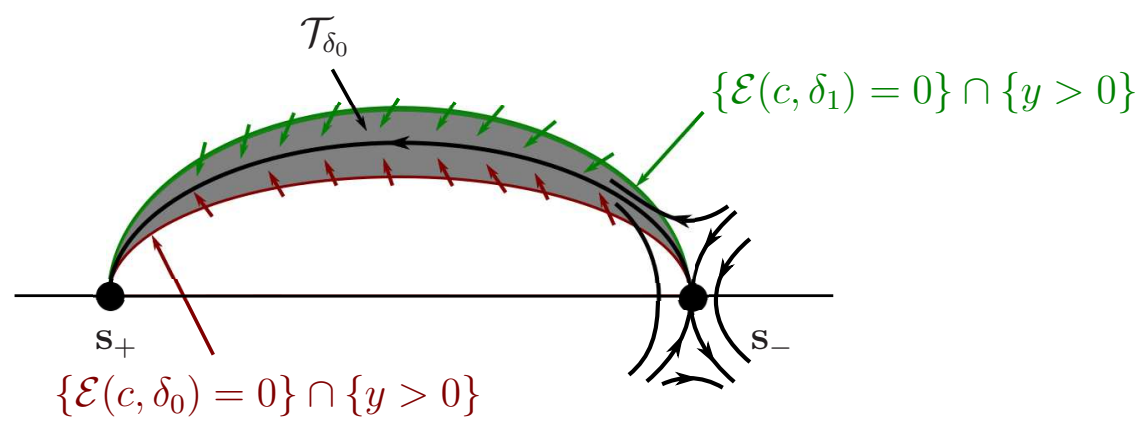

Fig. 17. Trapping region $\mathcal{T}_{\delta_{0}}$, see Proof of Proposition 8.

after removing the smaller ellipsoidal upper half disc

$$
\mathcal{B}_{\delta_{1}} \equiv\left\{1+c x+x^{2}+\delta_{1} y^{2}<0, y \geq 0\right\}
$$

from it, i.e.

$$
\mathcal{T}_{\delta_{0}}=\mathcal{B}_{\delta_{0}}^{*} \backslash \mathcal{B}_{\delta_{1}}
$$

Clearly by Lemma $3 \mathcal{T}_{\delta_{0}}$ is a trapping region that intersects the horizontal axis at $\mathbf{s}_{ \pm}$as drawn in Figure 17. Next we can choose $\delta_{0}>1$ such that the unstable trajectory $\mathcal{U}^{+}\left(\mathbf{s}_{-}\right) \backslash\left\{\mathbf{s}_{-}\right\}$lies inside $\mathcal{T}_{\delta_{0}}$ for $y$ sufficiently small. Indeed for $\delta>0$ the ellipse $1+c x+x^{2}+\delta y^{2}=0$ (resp. the trajectory $\mathcal{U}^{+}\left(\mathbf{s}_{-}\right) \backslash\left\{\mathbf{s}_{-}\right\}$) can locally near $\mathbf{s}_{-}$for $y>0$ be written as

$$
x=\frac{-c+\sqrt{c^{2}-4}}{2}-\frac{\delta}{2 \sqrt{c^{2}-4}} y^{2}+O\left(y^{4}\right)\left(\text { resp. } x=\frac{-c+\sqrt{c^{2}-4}}{2}+\kappa_{1-} y^{2}+O\left(y^{4}\right)\right) .
$$

If we take $\delta_{0}=\max \left\{1,-4 \sqrt{c^{2}-4} \kappa_{1-}\right\}$ then $0<\delta_{1}<-2 \sqrt{c^{2}-4} \kappa_{1-}<\delta_{0}$ by the identity $\delta_{1}=$ $2 \sqrt{c^{2}-4} \kappa_{1+}$ from Lemma 3 and since $\kappa_{1-}<-\kappa_{1+}$ for all $b>0$.

As $\mathcal{T}_{\delta_{0}}$ contains only the singularities $\mathbf{s}_{ \pm}$it then follows that $\mathcal{U}^{+}\left(\mathbf{s}_{-}\right) \backslash\left\{\mathbf{s}_{-}\right\}$converges to $\mathbf{s}_{+}$in positive time what we wanted to prove. The unicity follows by considering the two trapping regions formed by the closures of the connected components of $\mathcal{T}_{\delta_{0}} \backslash \mathcal{U}^{+}\left(\mathbf{s}_{-}\right)$.

Since $\delta_{1}(b, c)=\delta_{2}(b, c)$ on the curve $b=b_{-}(c)$, this argument does not apply for parameter values $\left(b_{-}(c), c\right)$. Using continuity with respect to the parameter $(b, c)$ the first case then is excluded too. Indeed in the first case we have that $\omega\left(\mathbf{s}_{-}\right)=\alpha\left(\mathbf{s}_{-}\right)$and hence also $\omega\left(\mathbf{s}_{+}\right)=\alpha\left(\mathbf{s}_{+}\right)$, where $\alpha\left(\mathbf{s}_{ \pm}\right)$and $\omega\left(\mathbf{s}_{ \pm}\right)$ refer to the traditional $\alpha$ - and $\omega$-limit sets of the singularities $\mathbf{s}_{ \pm}$. In particular there exists a period annulus surrounding the elliptic sector $\alpha\left(\mathbf{s}_{+}\right)$that is bounded by the homoclinic loop at $\mathbf{s}_{-}$in the half plane $\{x<0\}$. By continuity of the solutions with respect to the parameter and symmetry with respect to the $x$-axis there exists a period annulus surrounding the nilpotent singularity at $\mathbf{s}_{+}$in the half plane $\{x<0\}$ for small variations of the parameter. Therefore there exists a parameter $(b, c)$ with $b_{-}(c)-b>0$ sufficiently small having a period annulus in the half plane $\{x<0\}$. This is in contradiction with what we just proved for $(b, c)$ with $b<b_{-}(c)$. Therefore the global phase portrait along $b=b_{-}(c)$ is topologically equivalent to the one for $0<b<b_{-}(c)$.

\section{Acknowledgments}

The authors are partially supported by the MICIIN/FEDER grant number MTM2008-03437 and by the AGAUR grant number 2009SGR 410. Furthermore the first author also is supported by the Juan de la Cierva grant number JCI-2007-49-764 and the second author also is partially supported by ICREA Academia. 


\section{References}

Andreev, A. F. [1958] "Investigation of the behaviour of the integral curves of a system of two differential equations in the neighbourhood of a singular point," Amer. Math. Soc. Transl. (2) 8, 183-207.

Bautin, N. N. [1954] "On the number of limit cycles which appear with the variation of coefficients from an equilibrium position of focus or center type," American Math. Soc. Translation 1954, 19.

Blows, T. R. \& Rousseau, C. [1993] "Bifurcation at infinity in polynomial vector fields," J. Differential Equations 104, 215-242.

Caubergh, M., Llibre, J. \& Torregrosa, J. [2011a] "Global classification of a class of cubic vector fields whose canonical regions are period annuli," International Journal of Bifurcation and Chaos 21.

Caubergh, M., Llibre, J. \& Torregrosa, J. [2011b] "Global classification of a class of reversible cubic centers having noncollinear singularities," Preprint.

Dulac, H. [1908] "Détermination et integration d'une certaine classe d'équations différentielle ayant par point singulier un centre," Bull. Sci. Math. Sér. (2) 32, 230-252.

Dumortier, F., Llibre, J. \& Artés, J. C. [2006] Qualitative theory of planar differential systems, Universitext (Springer-Verlag, Berlin), ISBN 3-540-32893-9.

Hilbert, D. [1902] "Mathematical problems," Bull. Amer. Math. Soc. 8, 437-479, doi:10.1090/ S0002-9904-1902-00923-3, URL http://dx.doi.org/10.1090/S0002-9904-1902-00923-3.

Kapteyn, W. [1911] "On the midpoints of integral curves of differential equations of the first degree," Nederl. Akad. Wetensch. Verslag. Afd. Natuurk. Konikl. Nederland, 1446-1457.

Kapteyn, W. [1912] "New investigations on the midpoints of integrals of differential equations of the first degree," Nederl. Akad. Wetensch. Verslag Afd. Natuurk. 20, 1354-1365.

Schlomiuk, D. [1993] "Algebraic particular integrals, integrability and the problem of the center," Trans. Amer. Math. Soc. 338, 799-841.

Shi, S. L. [1980] "A concrete example of the existence of four limit cycles for plane quadratic systems," Sci. Sinica 23, 153-158.

Smale, S. [1998] "Mathematical problems for the next century," Math. Intelligencer 20, 7-15.

Vulpe, N. I. \& Sibirskiǔ, K. S. [1988] "Centro-affine invariant conditions for the existence of a center of a differential system with cubic nonlinearities," Dokl. Akad. Nauk SSSR 301, 1297-1301.

Żołądek, H. [1994a] "On a certain generalization of Bautin's theorem," Nonlinearity 7, 273-279.

Żołạdek, H. [1994b] "Quadratic systems with center and their perturbations," J. Differential Equations 109, 223-273. 